\title{
Compilação atualizada das espécies de morcegos (Chiroptera) para a Amazônia Brasileira
}

\author{
Enrico Bernard ${ }^{1,4}$, Valéria da Cunha Tavares $^{2}$ \& Erica Sampaio ${ }^{3}$ \\ ${ }^{1}$ Departamento de Zoologia, Centro de Ciências Biológicas, Universidade Federal de Pernambuco - UFPE, \\ Rua Nelson Chaves, s/n, Cidade Universitária, CEP 50670-420, Recife, PE, Brasil \\ ${ }^{2}$ Departamento de Zoologia, Instituto de Ciências Biológicas, Universidade Federal de Minas Gerais - UFMG, \\ Av. Antônio Carlos, 6627, CEP 31270-010, Belo Horizonte, MG, Brasil, e-mail: val.c.tavares@gmail.com \\ ${ }^{3}$ Institute of Experimental Ecology, University of Ulm, \\ Albert Einstein Allee, 11,D 89069,Ulm, Alemanha, e-mail: erica.sampaio@uni-ulm.de \\ ${ }^{4}$ Autor para correspondência: Enrico Bernard, e-mail: enrico.bernard@ufpe.br
}

BERNARD, E., TAVARES, V.C. \& SAMPAIO, E. Updated compilation of bat species (Chiroptera) for the Brazilian Amazonia. Biota Neotrop. 11(1): http://www.biotaneotropica.org.br/v11n1/en/abstract?article+bn00611012011.

\begin{abstract}
Brazil holds nearly 15\% of world's bat species richness, and most of it is present in the Amazonian biome. Here we present an updated compilation of bat species for the Brazilian Amazonia, with records of 146 species, belonging to nine families and 64 genera. At least 46 of those species are currently restricted to the Amazonian biome. Pará State is the species richest (120 spp.) and the Amazonian portion of Maranhão the least (21 spp.). Nine species were recorded in all the Amazonian States, and 28 are restricted to a single state. The species list for the region is not yet completed and is very likely that the Brazilian Amazonia holds more than 160 species of bats. We indicated priority areas for inventories in the region, and discuss some factors that can contribute to a further increase in the knwoledge of the regional bat fauna.
\end{abstract}

Keywords: Amazônia Legal, bat species richness, biodiversity inventories.

BERNARD, E., TAVARES, V.C. \& SAMPAIO, E. Compilação atualizada das espécies de morcegos (Chiroptera) para a Amazônia Brasileira. Biota Neotrop. 11(1): http://www.biotaneotropica.org.br/v11n1/pt/ abstract?article+bn00611012011.

Resumo: O Brasil abriga cerca de $15 \%$ da riqueza de morcegos do planeta, e grande parte desta riqueza está presente no bioma Amazônia. Apresentamos aqui uma compilação atualizada das espécies de morcegos da Amazônia Legal Brasileira, com registros formais de 146 espécies, distribuídas em nove famílias, e 64 gêneros. Pelo menos 46 destas espécies têm, até o momento, a ocorrência restrita ao bioma amazônico. O Pará é o estado com o maior número de espécies registradas (120), enquanto que a porção amazônica do Maranhão apresenta apenas 21 espécies. Nove espécies foram registradas em todos os estados amazônicos e 28 são conhecidas em apenas um estado. A lista de espécies de morcegos para a região não está esgotada e é bastante plausível que a Amazônia Brasileira contenha mais de 160 espécies de morcegos. Apontamos áreas prioritárias para a realização de inventários para morcegos na região, e discutimos alguns fatores que podem contribuir para o aumento do conhecimento da quiropterofauna amazônica.

Palavras-chave: Amazônia Legal, riqueza de espécies de morcegos, inventários de biodiversidade. 


\section{Introdução}

Morcegos apresentam uma grande importância ecológica, interagem com um amplo espectro de organismos, sendo dispersores de sementes, polinizadores, presas e predadores (e.g. Kunz \& Fenton 2003), têm um importante papel epidemiológico, incluindo a transmissão do vírus rábico (e.g. Schneider et al. 2009) e contribuem significativamente com a diversidade global dos mamíferos (Wilson \& Reeder 2005). Com 1150 espécies conhecidas, morcegos respondem por cerca de $25 \%$ da diversidade de mamíferos, sendo a segunda ordem com maior número de espécies, após os roedores (Simmons 2005). Comunidades de morcegos estão entre as mais ricas e ecologicamente diversas entre todas as comunidades de mamíferos. Frequentemente a diversidade $\alpha$ destas comunidades excede a totalidade de outras das outras ordens de mamíferos juntas (e.g. Kingston, 2009) e registros de 60, 70 e até 90 espécies de morcegos em uma única localidade neotropical não são raros (e.g. Simmons \& Voss 1998, Bernard \& Fenton 2002, Sampaio et al. 2003, Faria et al. 2006). A participação dos morcegos na fauna brasileira é expressiva: das cerca de 690 espécies de mamíferos atualmente conhecidas no país, pelo menos 167 são morcegos, pertencentes a nove famílias (Paglia et al. no prelo). Estes números indicam que o Brasil abriga cerca de $15 \%$ da riqueza de morcegos do planeta, sendo o segundo país com o maior número de espécies, após a Colômbia, com 178 (Alberico et al. 2000).

O conhecimento sobre a diversidade de espécies de morcegos no Brasil vem aumentando significativamente nos últimos 15 anos (e.g. Uieda \& Pedro 1996, Reis et al. 2007, Pacheco et al. 2008, Tavares et al. 2008), mas ainda é bastante heterogêneo. A distribuição espacial dos registros de morcegos no Brasil indica que menos de $10 \%$ do país pode ser considerado minimamente amostrado, e em cerca de $60 \%$ do território brasileiro não existe sequer um único registro formal de espécies de morcegos (Bernard et al. 2011). Apenas algumas poucas localidades já foram bem estudadas, e dispõem de listas de espécies próximas da real diversidade local (e.g. Bernard \& Fenton 2002, Sampaio et al. 2003, Faria et al. 2006). Entretanto, a maior parte do país permanece desconhecida com relação à sua fauna de morcegos (Bernard et al. 2011).

Cerca de $2 / 3$ do território brasileiro são cobertos pelo bioma Amazônia, e assim como para outros táxons, a Amazônia contribui com a maior parte da diversidade de espécies de morcegos do Brasil. Este é um reflexo da história evolutiva da região, da sua própria extensão e da diversidade de ambientes do bioma, que vão desde a floresta tropical chuvosa densa, até formações vegetais mais secas como os cerrados, as campinaranas, ou a vegetação inundável em sistemas de lagos rasos (e.g. Mittermeier et al. 2000). O bioma possui ainda importantes ecótonos com a Caatinga, na região nordeste do país, com o Cerrado, na região central, e com o Pantanal, na região centro-oeste.

Mesmo contribuindo com a maior parcela da diversidade de espécies, a Amazônia é uma enorme lacuna de conhecimento para a fauna de morcegos do Brasil. Existem registros formais de espécies de morcegos em menos de $24 \%$ do bioma Amazônia, contra cerca de $80 \%$ na Mata Atlântica, por exemplo (Bernard et al. 2011). A maior parte das coletas e registros na Amazônia está concentrada em poucas áreas, geralmente de acesso rápido e de fácil logística, próximas aos maiores centros urbanos, como Manaus, Belém, Santarém ou Macapá, ou ao longo de alguns dos grandes rios da região.

A Amazônia Brasileira constitui-se uma fronteira de ocupação social bastante dinâmica (e.g. Calentano \& Veríssimo 2007, Rodrigues et al. 2009) e porções significativas do bioma vêm experimentando alterações na sua integridade, com extensas áreas já desmatadas e outras sob forte pressão (e.g. Laurance et al. 2001, Morton et al. 2006, Hansen et al. 2008). Vivem na região cerca de
22 milhões de pessoas e atualmente a Amazônia concentra mais de $1 / 3$ do rebanho bovino brasileiro (IBGE 2010). Informar a sociedade sobre a riqueza e diversidade de espécies presente neste bioma é importante em vários sentidos: a) fornece subsídios para a tomada de decisões sobre o uso e destinação dos ambientes naturais, b) permite uma melhor avaliação dos impactos dos empreendimentos passados e futuros, c) permite uma melhor avaliação da qualidade dos estudos de impacto ambiental de empreendimentos que estão sendo realizados na região, d) indica lacunas de conhecimento, e e) aponta caminhos para pesquisas necessárias. Neste sentido, aqui é apresentada uma compilação atualizada das espécies de morcegos da Amazônia Brasileira, informando a riqueza conhecida por estados e famílias, apontando áreas prioritárias para a realização de inventários para morcegos na região, e indicando alguns fatores importantes para o aumento do conhecimento da quiropterofauna na região.

\section{Materiais e Métodos}

O ponto de partida para esta compilação foi uma listagem prévia, elaborada em 2004 e publicada somente quatro anos depois, na forma de um capítulo de um livro (Bernard \& Sampaio 2008). Infelizmente a publicação deste livro foi judicialmente suspensa e poucas pessoas tiveram acesso à informação nele contida. A lista de espécies de morcegos para a Amazônia Brasileira aqui apresentada contém correções e atualizações ocorridas desde 2004, e traz adições significativas à lista previamente publicada, que se encontra, além de embargada, já claramente defasada.

Foi adotada a definição política de Amazônia Legal, criada em 1953 (Lei 1.806, de 06.01.1953), e que engloba os Estados do Acre, Amapá, Amazonas, Pará, Rondônia, Roraima além da faixa do estado de Mato Grosso ao norte do paralelo $16^{\circ} \mathrm{S}$, a faixa ao norte do paralelo $13^{\circ} \mathrm{S}$ do então Estado de Goiás, hoje Tocantins, e a região a oeste do meridiano $44^{\circ} \mathrm{O}$ do Maranhão. Por envolver limites políticos bem definidos, os estados ou partes deles, esta definição da Amazônia Legal é apropriada, uma vez que permite que os registros sejam, em sua maior parte, separados por estados, municípios ou localidades de inventários.

Os registros foram compilados por meio de informações publicadas, na forma de artigos científicos em revistas indexadas, capítulos em livros, publicações especiais, revisões taxonômicas, relatórios técnicos e anais de congressos, ou ainda mediante registros de espécies em coleções brasileiras e estrangeiras. As coleções acessadas foram: Museu Paraense Emílio Goeldi (MPEG), em Belém, Pará; Instituto Nacional de Pesquisas da Amazônia (INPA), em Manaus, Amazonas; Museu de Zoologia da Universidade de São Paulo (MZUSP), em São Paulo; Museu Nacional, no Rio de Janeiro (MNRJ), no Rio de Janeiro; National Museum of Natural History (USNM), Smithsonian Institution, em Washington; American Museum of Natural History (AMNH), em Nova Iorque; The Field Museum (FMNH), em Chicago; Royal Ontario Museum (ROM), Ontário, Canadá; e British Museum (BM) em Londres, Inglaterra.

A lista aqui apresentada inclui registros para os estados do Acre (AC), Amapá (AP), Amazonas (AM), Pará (PA), Rondônia (RO) e Roraima (RR), e para as porções amazônicas do Maranhão (MA) e Mato Grosso (MT). Dados para o Estado do Tocantins, que são poucos, e não representam endemismo ou acréscimo ao número de espécies final, não foram incluídos em função da impossibilidade da precisa localização dos pontos de coleta, uma vez que, a maior parte deles está listada dentro do então Estado de Goiás. Até onde nosso conhecimento e informações de outros pesquisadores permitiram, foram evitados registros onde a identificação é considerada duvidosa. Há a possibilidade de um erro marginal na identificação de alguns registros, uma vez que parte das coletas de espécies de morcegos para a Amazônia não é acompanhada de material testemunho. 
Não é objetivo deste trabalho a solução dos diversos problemas taxônomicos que envolvem gêneros e espécies de morcegos com ocorrência na Amazônia Brasileira e, de forma a gerar uma lista para a região, frequentemente tivemos que decidir sobre a inclusão ou exclusão de espécies cujo status taxonômico encontra-se incerto. Estas decisões foram tomadas baseadas principalmente em revisões e na argumentação expressa em trabalhos publicados, na consulta direta a especialistas, ou ainda baseadas no nosso melhor discernimento. Neste processo de escolha, a nomenclatura dos registros aqui adotados seguiu Simmons (2005), com exceções de Vampyressa brocki e Vampyressa bidens, aqui tratadas como Vampyriscus (veja Baker et al. 2003), de Natalus, cujos registros previamente atribuídos a $N$. stramineus para o Brasil reconhecemos como N. espiritosantensis (Tejedor 2006, Tejedor et al. 2005), de Platyrrhinus, em função de significativas revisões recentes e descrições de novas espécies para o gênero (Velazco 2005, Velazco \& Gardner 2009, Velazco \& Patterson 2008, Velazco et al. 2010), e incluindo ainda Artibeus planirostris e não A. jamaicensis como espécie válida para a Amazônia Brasileira (Lim et al. 2004, Tavares et al. 2008), e Mimon cozumelae como sinônimo júnior de M. bennettii (mas veja Gregorin et al. 2008). Outros esclarecimentos sobre mudanças nomenclaturais desde Simmons (2005) podem ser obtidos em Gardner (2008) e Tavares et al. (2008).

\section{Resultados}

\section{Composição taxônomica}

A compilação corrigida e atualizada dos registros de espécies de morcegos para a Amazônia Brasileira indica a ocorrência de pelo menos 146 espécies, distribuídas em 64 gêneros (Tabela 1). Estão presentes as nove famílias de morcegos conhecidas para a Região Neotropical: Emballonuridae, Phyllostomidae, Mormoopidae, Noctilionidae, Furipteridae, Thyropteridae, Natalidae, Molossidae e Vespertilionidae. Phyllostomidae, com 80 espécies, é a família mais rica, seguida por Molossidae (24 espécies), Vespertilionidae (16), Emballonuridae (16, Mormoopidae (3), Thyropteridae (3), Noctilionidae (2), Natalidae e Furipteridae, com apenas uma espécie cada.

\section{Correções e atualizações}

Várias correções e adições foram feitas à lista de espécies previamente publicada para Amazônia Brasileira (Bernard \& Sampaio 2008). Pelo menos trinta e duas ocorrências foram acrescentadas à lista de espécies para o AP (Martins et al. 2006, Martins et al. no prelo). Entre os Emballonuridae, Dicliduris ingens foi registrado no MT (Bezerra \& Cunha 2007, Dalponte \& Aguiar 2009), e Peropteryx trinitatis incluído na lista do PA, baseado em Hood \& Gardner (2008). O registro de Pteronotus davyi (Mormoopidae) para o MT foi retirado da lista de espécies do bioma amazônico, pois segundo Gardner (2008), os exemplares de Cáceres (MT) identificados por Miranda-Ribeiro (1914) como $P$. davyi são na verdade $P$. gymnonotus, e Vieira (1955) considerou $P$. gymnonotus como um sinônimo júnior de P. davyi.

Entre os Phyllostomidae, o registro equivocado de Glossophaga longirostris para o AM (Bernard \& Sampaio 2008) foi corrigido. Incluímos o registro de Choeroniscus minor para o MT, baseado no registro de um exemplar em Alta Floresta (Gregorin \& Dietchfield 2005), e Choeroniscus godmani para o território brasileiro, baseado em um registro na Floresta Nacional do Tapajós, no PA (Presley et al. 2008). Carollia benkeithi (Solari \& Baker 2006) foi adicionado à lista de espécies da Amazônia e do Brasil, pois segundo McLellan \& Koopman (2008), espécimes previamente referidos para algumas localidades da Amazônia Brasileira como sendo Carollia castanea seriam exemplares de Carollia benkeithi. Desta forma, espécimes coletados no Brasil precisam ser reexaminados à luz da descrição de $C$. benkeithi, para determinar se uma, outra, ou ambas, ocorrem ou não no Brasil. Até que isso seja feito, mantivemos ambas as espécies como ocorrendo na região.

Revisões recentes para o gênero Platyrrhinus permitiram tanto a correção quanto a inclusão de novos registros para a região amazônica e para o território brasileiro. A espécie $P$. aurarius foi incluída na lista de espécies com registro no Brasil a partir de um exemplar coletado em Barcelos, AM (Velazco \& Gardner 2009). O mesmo ocorreu com $P$. fusciventris, a partir de registros no AM, AP e PA (Velazco et al. 2010). Segundo Velazco \& Patterson (2008), P. helleri não ocorre no Brasil, pois os exemplares sulamericanos assim designados, exceto P. matapalensis (descrita por Velazco 2005, para terras baixas do lado pacífico dos Andes, no Equador, Perú e Colombia), devem ser nomeados $P$. incarum. Desta forma, a designação $P$. helleri não deve então ser mais aplicada às populações sulamericanas (Velazco \& Patterson 2008). Mantivemos P. lineatus para o bioma amazônico baseado no registro de um exemplar na Serra do Roncador, $264 \mathrm{~km}$ ao Norte de Xavantina, e portanto dentro dos limites oficiais do bioma (Pine et al. 1970). Ressaltamos que especial atenção deve ser dada à confirmação de possíveis exemplares atribuídos a esta espécie oriundos de outras regiões mais ao norte deste registro.

Alteramos a designação do gênero Vampyressa para Vampyriscus no caso das espécies Vampyriscus bidens e Vampyriscus brocki (Baker \& Hoofer 2006), removendo ainda um registro errôneo desta última para RO. Baseado em observações de Gardner 2008, removemos também um registro errôneo de Vampyressa nymphea para o AM (McNab 1969), retirando assim esta espécie também da lista brasileira. Para RO foi acrescentado o registro de Vampyrum spectrum (Discher et al. 2009). Outra consideração importante refere-se à sinonimização de Micronycteris homezi com M. minuta, prevalecendo a segunda, como proposto por Ochoa \& Sánchez (2005). Estes autores rejeitam a fossa interauricular como principal característica de diferenciação da espécie, indicando que tal caracter também pode ser encontrado em alguns exemplares de M. megalotis and M. microtis. O registro de M. homezi foi mantido até que a sua validade seja melhor esclarecida e não reste dúvida sobre o status do táxon.

Atualizamos a designação da espécie de Natalidae para a América do Sul baseado nas revisões de Tejedor (2006) e Tejedor et al. (2005), que apontam que Natalus stramineus, anteriormente um táxon único ocorrente nas Américas Central, do Sul, nas Antilhas Maiores e Menores, é na verdade um conjunto de espécies, sendo três delas distribuídas nas Antilhas Maiores, uma espécie para a América Central, e uma para a América do Sul, ao Sul do Rio Amazonas. Esta última deve ser tratada como Natalus espiritosantensis, sendo o táxon $N$. stramineus agora restrito às Antilhas Menores.

O registro equivocado de Eptesicus fuscus para o AP em Bernard \& Sampaio (2008) foi corrigido, e consequentemente esta espécie foi retirada da lista amazônica. Embora Myotis nigricans deva ocorrer nestes estados, os registros desta espécie para MT, RO e RR foram retirados por falta de registros formais. Lasiurus castaneus teve sua ocorrência confirmada para o AM (Gardner 2008). O registro equivocado de Cynomops planirostris para o AM e PA foi corrigido, juntamente com a citação correta da referência desta espécie para o MT (Pine et al. 1970). Incluímos a ocorrência de Molossus currentium na região amazônica, nos Estados do AM e RO (López-González \& Presley 2001, Eger 2008). Entretanto, ressaltamos que esta ocorrência pode ser questionada em função do indivíduo analisado ser um jovem, o que dificulta a identificação inquestionável do taxon. 
Tabela 1. Famílias e espécies de morcegos registradas na Amazônia Legal Brasileira. Os números nas Referências seguem aqueles entre \{\} ao final de cada citação da seção Referências Bibliográficas. AC, Acre; AM, Amazonas; AP, Amapá; MA, porção amazônica do Maranhão; MT, porção amazônica do Mato Grosso; PA, Pará; RO, Rondônia; RR, Roraima; END, espécies endêmicas ao bioma Amazônia.

Table 1. Bat species and families currently recorded in the Amazônia Legal Brasileira. Reference numbers are those in \{\} at the end of each citation in the Reference section. AC, Acre; AM, Amazonas; AP, Amapá; MA, Amazonian portion of Maranhão; MT, Amazonian portion of Mato Grosso; PA, Pará; RO, Rondônia; RR, Roraima; END, species endemic to the Amazonian biome.

\begin{tabular}{|c|c|c|c|c|c|c|c|c|c|c|}
\hline Família/Espécie & $\mathbf{A C}$ & $\mathbf{A M}$ & $\mathbf{A P}$ & MA & MT & $\mathbf{P A}$ & RO & $\mathbf{R R}$ & END & Referências \\
\hline \multicolumn{11}{|l|}{ Emballonuridae } \\
\hline Centronycteris maximilliani (J. Fischer, 1829) & - & $\mathrm{X}$ & $\mathrm{X}$ & - & - & $\mathrm{X}$ & - & - & - & $7,75,105,107,113,114,125$ \\
\hline Cormura brevirostris Wagner, 1843 & - & $\mathrm{X}$ & - & - & $\mathrm{X}$ & $\mathrm{X}$ & $\mathrm{X}$ & - & $\mathrm{X}$ & $7,16,45,73,99,105,107,156,160$ \\
\hline Cyttarops alecto Thomas, 1913 & - & - & - & - & - & $\mathrm{X}$ & - & - & $\mathrm{X}$ & $53,141,153$ \\
\hline Diclidurus albus Wied-Neuwied, 1820 & - & $\mathrm{X}$ & $\mathrm{X}$ & - & - & $\mathrm{X}$ & $\mathrm{X}$ & - & - & $96,99,105,107,113,156$ \\
\hline Diclidurus ingens Hernandez-Camacho, 1955 & - & - & - & - & $\mathrm{X}$ & $\mathrm{X}$ & - & - & - & $9,14,23$ \\
\hline Diclidurus isabella Thomas, 1920 & - & $\mathrm{X}$ & - & - & - & - & - & - & $\mathrm{X}$ & 145 \\
\hline Diclidurus scutatus Peters, 1869 & - & $\mathrm{X}$ & $\mathrm{X}$ & - & - & $\mathrm{X}$ & - & - & $\mathrm{X}$ & $53,99,145$ \\
\hline Peropteryx leucoptera Peters, 1867 & - & $\mathrm{X}$ & - & - & - & $\mathrm{X}$ & - & - & - & $5,73,105,107,113,156$ \\
\hline Peropteryx kappleri Peters, 1867 & - & $\mathrm{X}$ & $\mathrm{X}$ & $\mathrm{X}$ & - & $\mathrm{X}$ & - & - & - & $76,83,84,99,105$ \\
\hline Peropteryx macrotis (Wagner, 1843) & - & $\mathrm{X}$ & $\mathrm{X}$ & $\mathrm{X}$ & $\mathrm{X}$ & $\mathrm{X}$ & - & - & - & $7,36,75,99,105,107,155,156,159$ \\
\hline Peropteryx trinitatis & - & - & - & - & - & $\mathrm{X}$ & - & - & - & 50 \\
\hline Rhynchonycteris naso (Wied-Neuwied, 1820) & $\mathrm{X}$ & $\mathrm{X}$ & $\mathrm{X}$ & - & $\mathrm{X}$ & $\mathrm{X}$ & $\mathrm{X}$ & $\mathrm{X}$ & - & $\begin{array}{l}7,19,45,75,84,87,97,99,105,108 \\
110,117,153,156,159\end{array}$ \\
\hline Saccopteryx bilineata (Temminck, 1838) & $\mathrm{X}$ & $\mathrm{X}$ & $\mathrm{X}$ & $\mathrm{X}$ & $\mathrm{X}$ & $\mathrm{X}$ & $\mathrm{X}$ & $\mathrm{X}$ & - & $\begin{array}{l}7,70,72,75,87,99,101,105,107 \\
110,115,117,155,156,159\end{array}$ \\
\hline Saccopteryx canescens Thomas, 1901 & - & $\mathrm{X}$ & $\mathrm{X}$ & $\mathrm{X}$ & - & $\mathrm{X}$ & $\mathrm{X}$ & - & $\mathrm{X}$ & $7,72,99,110,115,117,138,159$ \\
\hline Saccopteryx gymnura Thomas, 1901 & - & - & - & - & - & $\mathrm{X}$ & - & - & $\mathrm{X}$ & $54,99,138$ \\
\hline Saccopteryx leptura (Schreber, 1774) & $\mathrm{X}$ & $\mathrm{X}$ & $\mathrm{X}$ & $\mathrm{X}$ & $\mathrm{X}$ & $\mathrm{X}$ & $\mathrm{X}$ & $\mathrm{X}$ & - & $\begin{array}{l}7,87,99,101,105,107,108,110 \\
115,117,159\end{array}$ \\
\hline \multicolumn{11}{|l|}{ Noctilionidae } \\
\hline Noctilio albiventris Desmarest, 1818 & $\mathrm{X}$ & $\mathrm{X}$ & $\mathrm{X}$ & - & $\mathrm{X}$ & $\mathrm{X}$ & - & - & - & $87,107,108,159$ \\
\hline Noctilio leporinus (Linnaeus, 1758) & $\mathrm{X}$ & $\mathrm{X}$ & $\mathrm{X}$ & - & $\mathrm{X}$ & $\mathrm{X}$ & - & $\mathrm{X}$ & - & $\begin{array}{l}18,74,75,84,87,99,105,107,150 \\
153,154,155\end{array}$ \\
\hline \multicolumn{11}{|l|}{ Mormoopidae } \\
\hline Pteronotus gymnonotus Natterer, 1843 & - & $\mathrm{X}$ & - & - & $\mathrm{X}$ & $\mathrm{X}$ & - & $\mathrm{X}$ & - & $9,36,110,115$ \\
\hline Pteronotus parnellii (Gray, 1843) & - & $\mathrm{X}$ & $\mathrm{X}$ & - & $\mathrm{X}$ & $\mathrm{X}$ & $\mathrm{X}$ & $\mathrm{X}$ & - & $\begin{array}{l}7,9,69,75,101,105,107,108,110 \\
115,128,146,153,159\end{array}$ \\
\hline Pteronotus personatus (Wagner, 1843) & - & $\mathrm{X}$ & $\mathrm{X}$ & - & $\mathrm{X}$ & $\mathrm{X}$ & - & - & - & $69,97,99,110$ \\
\hline \multicolumn{11}{|l|}{ Phyllostomidae } \\
\hline Chrotopterus auritus (Peters, 1856) & $\mathrm{X}$ & $\mathrm{X}$ & $\mathrm{X}$ & - & $\mathrm{X}$ & $\mathrm{X}$ & - & - & - & $7,9,36,72,76,84,87,115,159$ \\
\hline Glyphonycteris daviesi Hill, 1964 & - & $\mathrm{X}$ & - & - & - & $\mathrm{X}$ & $\mathrm{X}$ & - & - & $9,73,102,115,159$ \\
\hline Glyphonycteris sylvestris Thomas, 1896 & - & $\mathrm{X}$ & $\mathrm{X}$ & - & - & $\mathrm{X}$ & - & $\mathrm{X}$ & - & $\begin{array}{l}7,9,45,73,76,84,96,99,110 \\
115,159\end{array}$ \\
\hline Lampronycteris brachyotis Dobson, 1878 & $\mathrm{X}$ & $\mathrm{X}$ & $\mathrm{X}$ & - & - & $\mathrm{X}$ & - & - & - & $9,67,76,115$ \\
\hline Lonchorhina aurita Tomes, 1863 & - & $\mathrm{X}$ & - & $\mathrm{X}$ & - & $\mathrm{X}$ & - & $\mathrm{X}$ & - & $33,84,110,116,156$ \\
\hline Lonchorhina inusitata Handley \& Ochoa, 1987 & - & - & - & - & - & - & $\mathrm{X}$ & - & $\mathrm{X}$ & 48 \\
\hline Lophostoma brasiliense Peters, 1866 & - & $\mathrm{X}$ & $\mathrm{X}$ & - & - & $\mathrm{X}$ & - & $\mathrm{X}$ & - & $9,72,76,84,110,115,159$ \\
\hline Lophostoma carrikeri J. A. Allen, 1910 & - & $\mathrm{X}$ & - & - & - & $\mathrm{X}$ & - & - & $\mathrm{X}$ & 9,115 \\
\hline Lophostoma schulzi (Genoways \& Williams, 1980) & - & $\mathrm{X}$ & $\mathrm{X}$ & - & - & $\mathrm{X}$ & - & - & $\mathrm{X}$ & $7,71,76,115$ \\
\hline Lophostoma silvicolum d'Orbigny, 1836 & $\mathrm{X}$ & $\mathrm{X}$ & $\mathrm{X}$ & - & $\mathrm{X}$ & $\mathrm{X}$ & $\mathrm{X}$ & $\mathrm{X}$ & - & $\begin{array}{l}8,9,45,70,72,84,87,105,106,107 \\
110,115,159\end{array}$ \\
\hline Macrophyllum macrophyllum (Schinz, 1821) & $\mathrm{X}$ & $\mathrm{X}$ & $\mathrm{X}$ & - & - & $\mathrm{X}$ & - & - & - & $69,84,87,97,105,107,108$ \\
\hline Micronycteris hirsuta Peters, 1869 & $\mathrm{X}$ & $\mathrm{X}$ & - & - & - & $\mathrm{X}$ & - & $\mathrm{X}$ & - & $7,9,73,84,87,96,115$ \\
\hline Micronycteris homezi Pirlot, 1967 & - & - & - & - & - & $\mathrm{X}$ & - & - & $\mathrm{X}$ & $6,76,113$ \\
\hline Micronycteris megalotis (Gray, 1842) & $\mathrm{X}$ & $\mathrm{X}$ & $\mathrm{X}$ & $\mathrm{X}$ & $\mathrm{X}$ & $\mathrm{X}$ & $\mathrm{X}$ & $\mathrm{X}$ & - & $\begin{array}{l}7,84,87,96,97,105,106,107,110 \\
115,153,157\end{array}$ \\
\hline Micronycteris microtis Miller, 1898 & - & $\mathrm{X}$ & $\mathrm{X}$ & - & - & $\mathrm{X}$ & - & - & - & $11,76,96,105,115,123,126$ \\
\hline Micronycteris minuta (Gervais, 1856) & $\mathrm{X}$ & $\mathrm{X}$ & $\mathrm{X}$ & - & $\mathrm{X}$ & $\mathrm{X}$ & - & $\mathrm{X}$ & - & $45,72,84,87,97,99,123$ \\
\hline Micronycteris schmidtorum Sanborn, 1935 & - & $\mathrm{X}$ & $\mathrm{X}$ & - & - & $\mathrm{X}$ & - & - & - & $7,9,76$ \\
\hline
\end{tabular}


Tabela 1. Continuação...

\begin{tabular}{|c|c|c|c|c|c|c|c|c|c|c|}
\hline Família/Espécie & $\mathbf{A C}$ & $\mathbf{A M}$ & $\mathbf{A P}$ & MA & MT & PA & RO & $\mathbf{R R}$ & END & Referências \\
\hline Mimon bennettii (Gray, 1838) & - & - & $\mathrm{X}$ & - & $\mathrm{X}$ & - & - & - & - & 2,75 \\
\hline Mimon crenulatum (E. Geoffroy, 1803) & - & $\mathrm{X}$ & $\mathrm{X}$ & - & $\mathrm{X}$ & $\mathrm{X}$ & $\mathrm{X}$ & $\mathrm{X}$ & - & $\begin{array}{l}7,45,70,84,97,101,105,107,108 \\
110,115\end{array}$ \\
\hline Neonycteris pusilla Sanborn, 1949 & - & $\mathrm{X}$ & - & - & - & $\mathrm{X}$ & - & - & $\mathrm{X}$ & 118, S. Marques-Aguiar (com. pess.) \\
\hline Phylloderma stenops Peters, 1865 & - & $\mathrm{X}$ & $\mathrm{X}$ & - & $\mathrm{X}$ & $\mathrm{X}$ & $\mathrm{X}$ & - & - & $7,9,45,69,72,76,84,99,115$ \\
\hline Phyllostomus discolor Wagner, 1843 & $\mathrm{X}$ & $\mathrm{X}$ & $\mathrm{X}$ & - & $\mathrm{X}$ & $\mathrm{X}$ & - & $\mathrm{X}$ & - & $\begin{array}{l}7,9,55,76,84,87,99,105,106,107 \\
110,115,156,159,160\end{array}$ \\
\hline Phyllostomus elongatus (E. Geoffroy, 1810) & $\mathrm{X}$ & $\mathrm{X}$ & $\mathrm{X}$ & - & $\mathrm{X}$ & $\mathrm{X}$ & $\mathrm{X}$ & $\mathrm{X}$ & - & $\begin{array}{l}7,8,45,69,70,72,84,87,99,105 \\
106,107,110,115,156,159\end{array}$ \\
\hline Phyllostomus hastatus (Pallas, 1767) & $\mathrm{X}$ & $\mathrm{X}$ & $\mathrm{X}$ & $\mathrm{X}$ & $\mathrm{X}$ & $\mathrm{X}$ & $\mathrm{X}$ & $\mathrm{X}$ & - & $\begin{array}{l}7,9,20,45,69,70,72,84,87,97,99 \\
105,106,107,110,115,159\end{array}$ \\
\hline Phyllostomus latifolius Thomas, 1901 & - & $\mathrm{X}$ & - & - & - & $\mathrm{X}$ & - & - & $\mathrm{X}$ & 31,115 \\
\hline Tonatia bidens Spix, 1823 & - & - & - & - & $\mathrm{X}$ & - & - & - & - & 168 \\
\hline Tonatia saurophilla Koopman \& Williams, 1951 & $\mathrm{X}$ & $\mathrm{X}$ & $\mathrm{X}$ & - & - & $\mathrm{X}$ & - & $\mathrm{X}$ & - & $9,76,87,110,115,159,168$ \\
\hline Trachops cirrhosus (Spix, 1823) & $\mathrm{X}$ & $\mathrm{X}$ & $\mathrm{X}$ & - & - & $\mathrm{X}$ & $\mathrm{X}$ & $\mathrm{X}$ & - & $\begin{array}{l}8,45,69,70,72,74,84,87,105,106 \\
107,110,115\end{array}$ \\
\hline Trinycteris nicefori (Sanborn, 1949) & $\mathrm{X}$ & $\mathrm{X}$ & $\mathrm{X}$ & - & $\mathrm{X}$ & $\mathrm{X}$ & - & $\mathrm{X}$ & - & $\begin{array}{l}45,72,76,84,87,105,106,107 \\
115,159\end{array}$ \\
\hline Vampyrum spectrum (Linnaeus, 1758) & $\mathrm{X}$ & $\mathrm{X}$ & $\mathrm{X}$ & - & $\mathrm{X}$ & $\mathrm{X}$ & $\mathrm{X}$ & - & - & $\begin{array}{l}9,26,84,87,96,99,105,106,107 \\
115,156\end{array}$ \\
\hline Anoura caudifer (Geoffroy, 1818) & $\mathrm{X}$ & $\mathrm{X}$ & $\mathrm{X}$ & - & $\mathrm{X}$ & $\mathrm{X}$ & - & - & - & $\begin{array}{l}75,84,87,97,105,107,115,116 \\
133,153,155,159\end{array}$ \\
\hline Anoura geoffroyi Gray, 1838 & - & - & $\mathrm{X}$ & - & $\mathrm{X}$ & $\mathrm{X}$ & - & - & - & 31,76 \\
\hline Choeroniscus minor (Peters, 1868) & $\mathrm{X}$ & $\mathrm{X}$ & $\mathrm{X}$ & - & $\mathrm{X}$ & $\mathrm{X}$ & $\mathrm{X}$ & $\mathrm{X}$ & - & $\begin{array}{l}39,45,70,74,76,84,87,105,106 \\
107,108,110,115,153,159\end{array}$ \\
\hline Choeroniscus godmani Thomas, 1903 & - & - & - & - & - & $\mathrm{X}$ & - & - & $\mathrm{X}$ & 104 \\
\hline Glossophaga longirostris Miller, 1898 & - & - & - & - & - & - & - & $\mathrm{X}$ & $\mathrm{X}$ & 161,162 \\
\hline Glossophaga soricina (Pallas, 1766) & $\mathrm{X}$ & $\mathrm{X}$ & $\mathrm{X}$ & $\mathrm{X}$ & $\mathrm{X}$ & $\mathrm{X}$ & $\mathrm{X}$ & $\mathrm{X}$ & - & $\begin{array}{l}17,18,20,45,64,70,73,74,80,87, \\
101,105,106,107,108,115,128, \\
145,153,156,159\end{array}$ \\
\hline Glossophaga commissarisi Gardner, 1962 & - & $\mathrm{X}$ & - & - & - & - & - & - & $\mathrm{X}$ & $31,163,164$ \\
\hline Lichonycteris obscura Thomas, 1895 & - & $\mathrm{X}$ & - & - & - & $\mathrm{X}$ & - & - & - & $7,69,84,105,107$ \\
\hline Scleronycteris ega Thomas, 1912 & - & $\mathrm{X}$ & - & - & - & $\mathrm{X}$ & - & - & $\mathrm{X}$ & $9,140,156$ \\
\hline Lionycteris spurelli Thomas, 1913 & - & $\mathrm{X}$ & $\mathrm{X}$ & - & - & $\mathrm{X}$ & - & - & - & $45,72,84,115,133$ \\
\hline Lonchophylla mordax Thomas, 1903 & - & - & - & - & - & $\mathrm{X}$ & - & - & - & 45,99 \\
\hline Lonchophylla thomasi J.A. Allen, 1904 & $\mathrm{X}$ & $\mathrm{X}$ & $\mathrm{X}$ & - & - & $\mathrm{X}$ & $\mathrm{X}$ & $\mathrm{X}$ & $\mathrm{X}$ & $\begin{array}{l}45,69,73,74,84,87,105,106,107 \\
110,115,129,159\end{array}$ \\
\hline Carollia benkeithi Solari \& Baker 2006 & $\mathrm{X}$ & - & - & - & $\mathrm{X}$ & $\mathrm{X}$ & $\mathrm{X}$ & - & $\mathrm{X}$ & 31,127 \\
\hline Carollia brevicauda Schinz, 1821 & $\mathrm{X}$ & $\mathrm{X}$ & $\mathrm{X}$ & - & $\mathrm{X}$ & $\mathrm{X}$ & $\mathrm{X}$ & - & - & $7,70,76,87,105,106,107,108,115$ \\
\hline Carollia castanea H. Allen, 1890 & $\mathrm{X}$ & $\mathrm{X}$ & - & - & $\mathrm{X}$ & $\mathrm{X}$ & $\mathrm{X}$ & - & $X$ & $84,87,94,103,147$ \\
\hline Carollia perspicillata (Linnaeus, 1758) & $\mathrm{X}$ & $\mathrm{X}$ & $\mathrm{X}$ & $\mathrm{X}$ & $\mathrm{X}$ & $\mathrm{X}$ & $\mathrm{X}$ & $\mathrm{X}$ & - & $\begin{array}{l}7,9,18,20,45,64,70,72,74,87 \\
106,107,115,116,128,153,154, \\
155,157,159\end{array}$ \\
\hline Rhinophylla fischerae Carter, 1966 & $\mathrm{X}$ & $\mathrm{X}$ & - & - & - & $\mathrm{X}$ & $\mathrm{X}$ & - & $\mathrm{X}$ & $9,69,70,72,84,87,99,108,159$ \\
\hline Rhinophylla pumilio (Peters, 1865) & $\mathrm{X}$ & $\mathrm{X}$ & $\mathrm{X}$ & - & $\mathrm{X}$ & $X$ & $\mathrm{X}$ & $\mathrm{X}$ & - & $\begin{array}{l}7,45,69,70,73,74,84,87,97,101 \\
106,107,110,115,159\end{array}$ \\
\hline Sturnira lilium (E. Geoffroy, 1810) & $\mathrm{X}$ & $\mathrm{X}$ & $\mathrm{X}$ & - & $\mathrm{X}$ & $\mathrm{X}$ & $\mathrm{X}$ & $\mathrm{X}$ & - & $\begin{array}{l}7,9,45,69,70,72,74,84,87,92 \\
97,101,106,107,108,110,115,159\end{array}$ \\
\hline Sturnira magna de la Torre, 1966 & $\mathrm{X}$ & - & - & - & - & - & - & - & $\mathrm{X}$ & 87 \\
\hline Sturnira tildae de la Torre, 1959 & $\mathrm{X}$ & $\mathrm{X}$ & $\mathrm{X}$ & - & $\mathrm{X}$ & $\mathrm{X}$ & $\mathrm{X}$ & $\mathrm{X}$ & - & $\begin{array}{l}9,45,69,70,72,73,74,76,84,87 \\
101,110,115,159\end{array}$ \\
\hline Ametrida centurio Gray, 1847 & - & $\mathrm{X}$ & $\mathrm{X}$ & - & $\mathrm{X}$ & $\mathrm{X}$ & - & - & $\mathrm{X}$ & $\begin{array}{l}7,9,69,73,74,84,97,101,106 \\
107,108,115\end{array}$ \\
\hline Artibeus anderseni Osgood, 1916 & $\mathrm{X}$ & $\mathrm{X}$ & - & - & - & $\mathrm{X}$ & $\mathrm{X}$ & $\mathrm{X}$ & $\mathrm{X}$ & $9,45,84,90,110,132$ \\
\hline Artibeus cinereus (Gervais, 1856) & $\mathrm{X}$ & $\mathrm{X}$ & $\mathrm{X}$ & $\mathrm{X}$ & $\mathrm{X}$ & $X$ & $\mathrm{X}$ & - & - & $\begin{array}{l}7,46,73,74,84,97,101,106,107 \\
115,159\end{array}$ \\
\hline
\end{tabular}


Tabela 1. Continuação...

\begin{tabular}{|c|c|c|c|c|c|c|c|c|c|c|}
\hline Família/Espécie & $\mathbf{A C}$ & $\mathbf{A M}$ & $\mathbf{A P}$ & MA & MT & $\mathbf{P A}$ & RO & $\mathbf{R R}$ & END & Referências \\
\hline Artibeus concolor Peters, 1865 & - & $\mathrm{X}$ & $\mathrm{X}$ & - & - & $\mathrm{X}$ & $\mathrm{X}$ & $\mathrm{X}$ & - & $\begin{array}{l}7,9,69,73,74,76,84,99,106,107 \\
108,110,115,159\end{array}$ \\
\hline Artibeus glaucus Thomas, 1893 & - & - & - & - & - & $\mathrm{X}$ & - & $\mathrm{X}$ & $\mathrm{X}$ & 31, E. Sampaio (com. pess.) \\
\hline Artibeus gnomus Handley, 1987 & - & $\mathrm{X}$ & $\mathrm{X}$ & - & $\mathrm{X}$ & $\mathrm{X}$ & $\mathrm{X}$ & - & - & $7,9,46,72,74,76,115,159$ \\
\hline Artibeus lituratus (Olfers, 1818) & $\mathrm{X}$ & $\mathrm{X}$ & $\mathrm{X}$ & - & $\mathrm{X}$ & $\mathrm{X}$ & $\mathrm{X}$ & $\mathrm{X}$ & - & $\begin{array}{l}9,46,72,74,84,99,106,107,108 \\
110,115,132,159\end{array}$ \\
\hline Artibeus planirostris (Leach, 1821) & $\mathrm{X}$ & $\mathrm{X}$ & $\mathrm{X}$ & $\mathrm{X}$ & $\mathrm{X}$ & $\mathrm{X}$ & $\mathrm{X}$ & $\mathrm{X}$ & - & $7,46,74,84,106,107,132,159$ \\
\hline Artibeus obscurus (Schinz, 1821) & $\mathrm{X}$ & $\mathrm{X}$ & $\mathrm{X}$ & - & $\mathrm{X}$ & $\mathrm{X}$ & $\mathrm{X}$ & $\mathrm{X}$ & - & $\begin{array}{l}7,9,46,72,74,84,97,115,128 \\
132,159\end{array}$ \\
\hline Chiroderma trinitatum Goodwin, 1958 & $\mathrm{X}$ & $\mathrm{X}$ & $\mathrm{X}$ & - & $\mathrm{X}$ & $\mathrm{X}$ & - & - & $\mathrm{X}$ & $9,45,76,101,106,107,115,132$ \\
\hline Chiroderma villosum Peters, 1860 & $\mathrm{X}$ & $\mathrm{X}$ & $\mathrm{X}$ & - & $\mathrm{X}$ & $\mathrm{X}$ & $\mathrm{X}$ & $\mathrm{X}$ & - & $\begin{array}{l}7,9,44,45,69,76,101,128,132 \\
145,159\end{array}$ \\
\hline Enchisthenes hartii Thomas, 1892 & - & - & - & - & - & - & $\mathrm{X}$ & - & $\mathrm{X}$ & 70 \\
\hline Mesophylla macconnelli Thomas, 1901 & $\mathrm{X}$ & $\mathrm{X}$ & $\mathrm{X}$ & - & $\mathrm{X}$ & $\mathrm{X}$ & $\mathrm{X}$ & $\mathrm{X}$ & $\mathrm{X}$ & $\begin{array}{l}7,9,45,70,72,87,97,101,106,107 \\
110,115,159\end{array}$ \\
\hline Platyrrhinus aurarius Handley \& Ferris, 1972 & - & $\mathrm{X}$ & - & - & - & - & - & - & $\mathrm{X}$ & 150 \\
\hline Platyrrhinus brachycephalus Rouk \& Carter, 1972 & $\mathrm{X}$ & $\mathrm{X}$ & $\mathrm{X}$ & - & - & $\mathrm{X}$ & - & - & $\mathrm{X}$ & $9,47,73,74,76,132,149$ \\
\hline $\begin{array}{l}\text { Platyrrhinus fusciventris Velazco, Gardner \& } \\
\text { Patterson } 2010\end{array}$ & - & $\mathrm{X}$ & $\mathrm{X}$ & - & - & $\mathrm{X}$ & - & - & $\mathrm{X}$ & 152 \\
\hline Platyrrhinus incarium (Thomas, 1912) & $\mathrm{X}$ & $\mathrm{X}$ & $\mathrm{X}$ & - & $\mathrm{X}$ & $\mathrm{X}$ & $\mathrm{X}$ & - & - & $\begin{array}{l}9,45,69,70,72,73,74,97,101,106, \\
107,115,131,151,159\end{array}$ \\
\hline Platyrrhinus infuscus Peters, 1880 & $\mathrm{X}$ & $\mathrm{X}$ & - & - & - & - & - & - & $\mathrm{X}$ & $79,86,87,132,153,156$ \\
\hline Platyrrhinus lineatus (Geoffroy, 1810) & - & - & $\mathrm{X}$ & - & $\mathrm{X}$ & - & - & - & - & 76,101 \\
\hline Sphaeronycteris toxophyllum Peters, 1882 & $\mathrm{X}$ & $X$ & - & - & - & - & $\mathrm{X}$ & - & $\mathrm{X}$ & $31,95,99$ \\
\hline Uroderma bilobatum Peters, 1866 & $\mathrm{X}$ & $\mathrm{X}$ & $\mathrm{X}$ & - & $\mathrm{X}$ & $\mathrm{X}$ & $\mathrm{X}$ & $\mathrm{X}$ & - & $\begin{array}{l}7,9,25,45,69,70,72,74,97,99 \\
101,106,107,115,128,132,159\end{array}$ \\
\hline Uroderma magnirostrum Davis, 1968 & $\mathrm{X}$ & $\mathrm{X}$ & $\mathrm{X}$ & - & $\mathrm{X}$ & $\mathrm{X}$ & - & $\mathrm{X}$ & - & $\begin{array}{l}9,25,69,73,74,97,101,106,107 \\
108,128,132,159\end{array}$ \\
\hline Vampyriscus bidens Dobson, 1878 & $\mathrm{X}$ & $\mathrm{X}$ & $\mathrm{X}$ & - & - & $\mathrm{X}$ & $\mathrm{X}$ & - & $\mathrm{X}$ & $7,9,45,69,73,74,87,97,115$ \\
\hline Vampyriscus brocki Peterson, 1968 & - & $\mathrm{X}$ & - & - & - & $\mathrm{X}$ & - & - & $\mathrm{X}$ & 31,159 \\
\hline Vampyressa thyone Thomas, 1909 & $\mathrm{X}$ & - & $\mathrm{X}$ & - & - & - & $\mathrm{X}$ & - & $\mathrm{X}$ & $70,75,87$ \\
\hline Vampyrodes caraccioli (Thomas, 1889) & $\mathrm{X}$ & - & $\mathrm{X}$ & - & - & $\mathrm{X}$ & - & - & - & $76,99,132,145,156$ \\
\hline Desmodus rotundus (E. Geoffroy, 1810) & $\mathrm{X}$ & $\mathrm{X}$ & $\mathrm{X}$ & $\mathrm{X}$ & $\mathrm{X}$ & $\mathrm{X}$ & $\mathrm{X}$ & $\mathrm{X}$ & - & $\begin{array}{l}7,18,20,46,70,74,87,97,99,106 \\
107,108,115,120,128,159\end{array}$ \\
\hline Diaemus youngi (Jentink, 1893) & $\mathrm{X}$ & $\mathrm{X}$ & $\mathrm{X}$ & - & $\mathrm{X}$ & $\mathrm{X}$ & - & - & - & $7,45,72,87,97$ \\
\hline Diphylla ecaudata (Spix, 1823) & $\mathrm{X}$ & - & $\mathrm{X}$ & - & - & $\mathrm{X}$ & $\mathrm{X}$ & - & - & $31,87,97,99,156,159$ \\
\hline \multicolumn{11}{|l|}{ Natalidae } \\
\hline Natalus espiritosantensis (Ruschi, 1951) & - & - & - & - & $\mathrm{X}$ & $\mathrm{X}$ & - & - & - & $130,135,136,146$ \\
\hline \multicolumn{11}{|l|}{ Furipteridae } \\
\hline Furipterus horrens F. Cuvier, 1828 & - & $\mathrm{X}$ & - & - & - & $\mathrm{X}$ & - & - & - & 99,115 \\
\hline \multicolumn{11}{|l|}{ Thyropteridae } \\
\hline Thyroptera discifera Lichtenstein and Peters, 1855 & - & $\mathrm{X}$ & - & - & $\mathrm{X}$ & $\mathrm{X}$ & - & - & - & $99,100,115$ \\
\hline Thyroptera tricolor Spix, 1823 & $\mathrm{X}$ & $\mathrm{X}$ & $\mathrm{X}$ & - & - & $\mathrm{X}$ & - & - & - & $7,18,87,99,106,107,108,115$ \\
\hline Thyroptera lavali Pine, 1993 & - & - & - & - & - & $\mathrm{X}$ & - & - & $\mathrm{X}$ & 9,73 \\
\hline \multicolumn{11}{|l|}{ Vespertilionidae } \\
\hline Eptesicus andinus J. A. Allen, 1914 & - & - & - & - & $\mathrm{X}$ & - & - & - & $\mathrm{X}$ & 101 \\
\hline Eptesicus brasiliensis (Desmarest, 1819) & - & $\mathrm{X}$ & $\mathrm{X}$ & - & $\mathrm{X}$ & $\mathrm{X}$ & $\mathrm{X}$ & - & - & $24,31,99,144$ \\
\hline Eptesicus chiriquinus Thomas, 1920 & - & $\mathrm{X}$ & - & - & - & $\mathrm{X}$ & - & - & $\mathrm{X}$ & $9,31,54,125$ \\
\hline Eptesicus diminutus Osgood, 1915 & - & - & - & $\mathrm{X}$ & - & - & - & - & - & 165 \\
\hline Eptesicus furinalis (d'Orbigny, 1847) & - & $\mathrm{X}$ & $\mathrm{X}$ & - & $\mathrm{X}$ & $\mathrm{X}$ & - & - & - & $31,84,99,101$ \\
\hline Histiotus velatus I. Geoffroy, 1824 & - & - & - & $\mathrm{X}$ & $\mathrm{X}$ & - & - & - & - & 143,156 \\
\hline Lasiurus blossevillii (Lesson and Garnot, 1826) & - & $\mathrm{X}$ & $\mathrm{X}$ & - & $\mathrm{X}$ & $\mathrm{X}$ & - & - & - & $7,9,31,44,101,105,107,139$ \\
\hline Lasiurus castaneus Handley, 1960 & - & $\mathrm{X}$ & - & - & - & - & - & - & $\mathrm{X}$ & 31 \\
\hline Lasiurus ega Gervais, 1856 & $\mathrm{X}$ & $\mathrm{X}$ & - & - & $\mathrm{X}$ & $\mathrm{X}$ & - & - & - & $34,44,87,101$ \\
\hline Lasiurus egregius Peters, 1870 & - & - & - & - & - & $\mathrm{X}$ & - & - & - & 54,167 \\
\hline
\end{tabular}


Tabela 1. Continuação...

\begin{tabular}{|c|c|c|c|c|c|c|c|c|c|c|}
\hline Família/Espécie & $\mathrm{AC}$ & $\mathbf{A M}$ & $\mathbf{A P}$ & MA & MT & $\mathbf{P A}$ & RO & $\mathbf{R R}$ & END & Referências \\
\hline Myotis albescens (Geoffroy, 1806) & $\mathrm{X}$ & $\mathrm{X}$ & $\mathrm{X}$ & - & $\mathrm{X}$ & $\mathrm{X}$ & $\mathrm{X}$ & - & - & 87,156 \\
\hline Myotis nigricans (Schinz, 1821) & - & $\mathrm{X}$ & $\mathrm{X}$ & - & - & $\mathrm{X}$ & - & - & - & $97,99,115$ \\
\hline Myotis riparius (Handley, 1960) & $\mathrm{X}$ & $\mathrm{X}$ & $\mathrm{X}$ & - & - & $\mathrm{X}$ & - & - & - & $45,59,76,87,115$ \\
\hline Myotis simus Thomas, 1901 & - & $\mathrm{X}$ & - & - & $\mathrm{X}$ & $\mathrm{X}$ & - & - & - & 31,59 \\
\hline Rhogeessa hussoni Genoways and Baker, 1996 & - & - & - & $\mathrm{X}$ & - & - & - & - & - & 32 \\
\hline Rhogeessa io Thomas, 1903 & - & $\mathrm{X}$ & - & $\mathrm{X}$ & $\mathrm{X}$ & - & - & - & $\mathrm{X}$ & 60,84 \\
\hline \multicolumn{11}{|l|}{ Molossidae } \\
\hline Cynomops abrasus Temminck, 1827 & - & $\mathrm{X}$ & - & - & $\mathrm{X}$ & $\mathrm{X}$ & - & - & - & $101,115,153,166$ \\
\hline Cynomops greenhalli Goodwin, 1958 & - & $\mathrm{X}$ & - & - & - & - & - & - & - & 8 \\
\hline Cynomops paranus Thomas, 1901 & - & $\mathrm{X}$ & - & - & $\mathrm{X}$ & $\mathrm{X}$ & - & - & $\mathrm{X}$ & 9,31,98, 137, R. Gregorin (com. pess.) \\
\hline Cynomops planirostris Peters, 1865 & - & - & - & - & $\mathrm{X}$ & - & - & - & - & 101 \\
\hline Eumops auripendulus Shaw, 1800 & $\mathrm{X}$ & $\mathrm{X}$ & - & - & $\mathrm{X}$ & $\mathrm{X}$ & - & - & - & 28,87 \\
\hline Eumops bonariensis Peters, 1874 & - & $\mathrm{X}$ & - & - & - & $\mathrm{X}$ & - & - & - & 9,28 \\
\hline Eumops glaucinus Wagner, 1843 & - & $\mathrm{X}$ & - & - & $\mathrm{X}$ & $X$ & - & - & - & $\begin{array}{l}28,94,99,155,156, \\
\text { R. Gregorin (com. pess.) }\end{array}$ \\
\hline Eumops hansae Sanborn, 1932 & - & $\mathrm{X}$ & - & - & - & - & - & - & - & $28,31,43$ \\
\hline Eumops perotis Schinz, 1821 & - & $\mathrm{X}$ & - & $\mathrm{X}$ & - & $\mathrm{X}$ & - & - & - & $16,28,99,116$ \\
\hline Eumops trumbulli (Thomas, 1901) & - & $\mathrm{X}$ & $\mathrm{X}$ & - & - & $\mathrm{X}$ & - & - & $\mathrm{X}$ & $\begin{array}{l}28,97,99,103,108,141,153, \\
154,156\end{array}$ \\
\hline Molossops neglectus Williams \& Genoways, 1980 & - & - & - & - & - & $\mathrm{X}$ & - & - & - & 3 \\
\hline Molossops temminckii Burmeister, 1854 & - & $\mathrm{X}$ & - & - & $\mathrm{X}$ & - & - & - & - & $2,101,155,156,158$ \\
\hline Molossus barnesi Thomas, 1905 & - & - & - & - & - & $\mathrm{X}$ & - & - & - & 19 \\
\hline Molossus coibensis J. A. Allen, 1904 & - & - & - & - & $\mathrm{X}$ & - & - & - & $\mathrm{X}$ & $2,27,31$ \\
\hline Molossus currentium Thomas, 1901 & - & $\mathrm{X}$ & - & - & - & $\mathrm{X}$ & - & - & $\mathrm{X}$ & 31,65 \\
\hline Molossus molossus (Pallas, 1766) & $\mathrm{X}$ & $\mathrm{X}$ & $\mathrm{X}$ & $\mathrm{X}$ & $\mathrm{X}$ & $\mathrm{X}$ & $\mathrm{X}$ & $\mathrm{X}$ & - & $\begin{array}{l}2,27,69,87,97,99,101,107,128, \\
153,157\end{array}$ \\
\hline Molossus pretiosus Miller, 1902 & - & - & - & - & $\mathrm{X}$ & - & - & - & $\mathrm{X}$ & 40 \\
\hline Molossus rufus E. Geoffroy, 1805 & - & $\mathrm{X}$ & $\mathrm{X}$ & $\mathrm{X}$ & $X$ & $\mathrm{X}$ & - & - & - & $18,20,69,97,99,101,107$ \\
\hline Neoplatymops mattogrossensis Vieira, 1942 & - & - & - & - & $\mathrm{X}$ & $\mathrm{X}$ & $\mathrm{X}$ & - & - & 38 \\
\hline Nyctinomops laticaudatus (E. Geoffroy, 1805) & - & $\mathrm{X}$ & $\mathrm{X}$ & - & $\mathrm{X}$ & $\mathrm{X}$ & $\mathrm{X}$ & $\mathrm{X}$ & - & 38, R. Gregorin (com. pess.) \\
\hline Nyctinomops macrotis Gray, 1840 & - & - & - & $\mathrm{X}$ & $\mathrm{X}$ & $\mathrm{X}$ & - & - & - & 31, R. Gregorin (com. pess.) \\
\hline Promops centralis Thomas, 1915 & $\mathrm{X}$ & - & - & - & - & $\mathrm{X}$ & - & - & $\mathrm{X}$ & 40,87 \\
\hline Promops nasutus (Spix, 1823) & - & $\mathrm{X}$ & $\mathrm{X}$ & - & - & $\mathrm{X}$ & - & - & - & $80,97,142,153,156$ \\
\hline Tadarida brasiliensis I. Geoffroy, 1824 & - & - & - & - & - & - & - & $\mathrm{X}$ & - & 110 \\
\hline Total: 146 espécies & 59 & 110 & 79 & 21 & 74 & 120 & 50 & 42 & 46 & \\
\hline
\end{tabular}

\section{Distribuição das espécies}

O PA é o estado com o maior número de espécies registradas (120), seguido pelo AM (110), AP (79), MT (74), AC (59), RO (50), RR (42), e a porção amazônica do MA, com 21 espécies. Pelo menos cinco espécies aguardam confirmação para o AP (Cynomops cf. planirostris, Carollia cf. castanea, Glossophaga $\mathrm{cf}$. longirostris, Micronycteris cf. homezi, e Eptesicus cf. chiriquinus Martins et al., no prelo) o que pode elevar o número real de espécies para aquele estado. Nove espécies são amplamente distribuídas, com registros em todos os estados amazônicos: Saccopteryx bilineata e S. leptura, Micronycteris megalotis, Phyllostomus hastatus, Glossophaga soricina, Carollia perspicillata, Artibeus planirostris, Desmodus rotundus e Molossus molossus. De maneira contrária, existe um grupo de 28 espécies cujos registros são conhecidos em apenas um estado: Cyttarops alecto (PA), Diclidurus isabella (AM), Peropteryx trinitatis (PA), Saccopteryx gymnura (PA), Lonchorhina inusitata (RO), Micronycteris homezi (PA), Tonatia bidens (MT), Choeroniscus godmani (PA),
Glossophaga longirostris (RR), Glossophaga commissarisi (AM), Lonchophylla mordax (PA), Sturnira magna (AC), Enchisthenes hartii (RO), Platyrrhinus aurarius (AM), Thyroptera lavali (PA), Eptesicus andinus (MT), Eptesicus diminutus (MA), Lasiurus castaneus (AM), Lasiurus egregius (PA), Rhogeessa hussoni (MA), Cynomops greenhalli (AM), Cynomops planirostris (MT), Eumops hansae (AM), Molossops neglectus (PA), Molossus barnesi (PA), Molossus coibensis (MT), Molossus pretiosus (MT), e Tadarida brasiliensis (RR) (Tabela 1).

\section{Discussão}

\section{Riqueza de espécies e distribuição do conhecimento}

Considerando 167 espécies para o Brasil (Paglia et al. no prelo), com registros de pelo menos 146 espécies a Amazônia Legal abriga $87 \%$ da fauna de morcegos conhecida para o país, confirmando a importância do bioma para a diversidade nacional, não apenas para morcegos, mas para mamíferos em geral. Esta porcentagem 
é superior quando comparada à de outros grupos de animais. Por exemplo, a Amazônia tem cerca de $76 \%$ das espécies de aves, $42 \%$ das espécies de cobras e 39\% das espécies de lagartos e anfisbenídeos conhecidas no país (Marini \& Garcia 2005, Rodrigues 2005). De fato, a significativa contribuição dos morcegos na riqueza faunística da Amazônia deve-se em parte ao elevado número de espécies aparentemente endêmicas à região: das 146 espécies registradas, pelo menos 46 tem, até o momento, a ocorrência restrita ao bioma amazônico (Tabela 1).

A lista de espécies de morcegos para a Amazônia Brasileira não está esgotada. Todos os países limítrofes à região possuem registros de espécies cuja ocorrência é bastante provável para o território brasileiro. Na Guiana Francesa, por exemplo, são seis espécies nesta condição: Micronycteris brosseti, Natalus tumidirostris, Lasiurus atratus, Eumops maurus, e Molossus sinaloe (Best et al. 2001, Catzeflis 2010); no Suriname, Thyroptera devivoi; e na Guyana, Anoura latidens e Vampyressa pusilla (Lim et al. 2005). Registros de Lonchorhina orinocensis, Histiotus humboldti e Myotis oxyotus na Venezuela, de Lonchorhina marinkellei na Colômbia, de Micronycteris matses no Perú, e de Chiroderma salvini na Bolívia, todos próximas à fronteira com o Brasil (veja Gardner 2008), podem indicar a possibilidade de ocorrência destas espécies também na Amazônia Brasileira. Situação similar ocorre com a presença do gênero Mormoops, uma vez que há registros para a espécie na Guyana, a cerca de $300 \mathrm{~km}$ da fronteira com o Brasil (Gardner 2008). Há ainda o caso de espécies com registros próximas à faixa de transição entre a Amazônia e o Cerrado no Brasil, tais como Glyphonycteris behnii e Chiroderma doriae (Sanborn 1949, Carter \& Dolan 1978, Gregorin 1998). Além disso, há ainda novas espécies que se encontram em processo de descrição e a aceleração da revisão de material proveniente da Amazônia tombado em museus pode indicar novos registros. A situação do gênero Platyrrhinus, onde revisões recentes alteraram bastante o seu status taxonômico (veja Velazco et al. 2010), é um bom exemplo. Desta forma, é bastante plausível que a Amazônia Brasileira contenha mais de 160 espécies de morcegos.

O conhecimento sobre a ocorrência de espécies na Amazônia é, sobretudo, heterogêneo, com poucas áreas relativamente bem estudadas e grandes extensões que nunca foram amostradas. Considerando-se a extensão dos biomas brasileiros, a Amazônia é o menos amostrado para morcegos (Bernard et al. 2011). Para mamíferos em geral, Silva et al. (2001) constataram que, baseado nas coleções do INPA, MPEG, AMNH e FMNH, mais de $85 \%$ dos registros existentes são provenientes de apenas uma ou até no máximo 10 localidades por bacia hidrográfica ou estado, e ainda que, em média, menos de 50 espécies de mamíferos são registradas por localidade amostrada, sendo que os valores mais elevados estão associados a pesquisas que incluem a coleta de morcegos.

A heterogeneidade na riqueza de espécies registrada entre os estados da Amazônia Legal reflete claramente a importância da existência de centros de pesquisa com um histórico de coleta a eles associado. Pará e AM contam com cerca do dobro ou mais de espécies registradas no $\mathrm{AC}, \mathrm{RO}$ ou $\mathrm{RR}$, e isto se deve em grande parte à presença de instituições de pesquisa consolidadas nestes estados, como o INPA, em Manaus, e o MPEG, em Belém. Ambas as instituições contam com coleções de referência para morcegos, e suportam programas de pós-graduação e pesquisa, influenciando direta e positivamente no conhecimento científico da fauna local. Entretanto, mesmo assim, o conhecimento para o PA e AM ainda pode ser bastante melhorado.

No AP, o estabelecimento de um grupo de pesquisas em morcegos no Instituto de Pesquisas Científicas e Tecnológicas (IEPA) propiciou um salto significativo no conhecimento sobre a diversidade e distribuição dos morcegos no estado, partindo de
48 espécies registradas até 2006, para 79 espécies confirmadas após menos de dois anos de pesquisas e inventários (E. Bernard comunicação pessoal). Assim, além de apoiar um aumento e fixação de pesquisadores trabalhando com morcegos na região como um todo, especial atenção deve ser dada aos estados que contam com listas claramente sub-amostradas, de forma a preencher as lacunas de informação de maneira mais espacialmente uniforme e diminuir as discrepâncias sobre o conhecimento da fauna de morcegos na região.

\section{2. Áreas prioritárias para futuros inventários}

Bernard et al. (2011) computaram a distribuição dos registros conhecidos de morcegos no Brasil e agruparam-nos em células de 0,5 graus de latitude $\times 0,5$ graus de longitude (cada uma com cerca de $3000 \mathrm{~km}^{2}$ ). Esta análise indica que, embora conte com as localidades com a maior riqueza de espécies do país, apenas $23,9 \%$ da Amazônia dispõem de pelo menos um único registro formal para o grupo, e somente $5,1 \%$ do bioma podem ser considerados minimamente amostrados, contando com listas com no mínimo 20 espécies. É muito pouco diante da extensão, riqueza biológica e importância deste bioma.

A distribuição fragmentada dos registros disponíveis para espécies de morcegos na Amazônia Brasileira indica que ainda existem grandes lacunas de conhecimento que precisam ser preenchidas. Estas lacunas são: as porções amazônicas do TO, MA e MT; o Sul do PA e o Noroeste da Calha Norte Paraense; o Centro-Sul de RO; as porções Norte e Sul do AM distantes da capital Manaus; a porção Norte do AP; e a porção Centro-Norte de RR.

Podem ainda ser adicionadas a estas áreas, outras quatro apontadas por Silva et al. (2001) como prioritárias para a realização de inventários gerais na Amazônia:

- As áreas de transição vegetal entre o Bioma Amazônico e o Cerrado, Pantanal e a Caatinga;

- As matas de bambu, campinaranas, e enclaves de cerrado nas bordas das florestas de dossel fechado, e buritizais do oeste da Amazônia;

- O Sudeste da Amazônia, uma região extremamente degradada em muitas áreas e com importantes remanescentes; e

- As áreas ao longo do eixo Solimões-Amazonas e nos cursos baixos e médios dos rios da região, onde existe alta heterogeneidade de hábitats em contato.

\section{Chaves de identificação}

Identificar corretamente um organismo é essencial para o seu registro fidedigno. A grande riqueza de espécies de morcegos e a semelhança entre várias delas, em muitos casos distinguíveis apenas por caracteres cranianos ou genéticos, representam uma dificuldade real para a correta identificação e registro das espécies de morcegos para a Amazônia Brasileira. Tal fato é agravado por não existir, até o momento, uma chave de identificação especificamente desenvolvida para as espécies da Amazônia brasileira. As identificações atuais baseiam-se ou em chaves já defasadas, ou em chaves mais abrangentes para morcegos da América do Sul (Gardner 2008), ou áreas mais restritas, como as Guianas (Lim \& Engstrom 2001). São usadas ainda chaves restritas a famílias, como Molossidae (Gregorin \& Taddei 2002), ou ainda gêneros como, por exemplo, Sturnira (Giannini \& Barquez 2003), Diclidurus (Ceballos \& Medellín 1988), Micronycteris (Simmons 1996), Lasiurus (Shump Jr. \& Shump 1982), Lonchorhina (Lassieur \& Wilson 1989), Mimon (Ortega \& Arita 1997) e Platyrrhinus (Ferrell \& Wilson 1991). Neste sentido, é necessária uma ampla revisão dos caracteres presentes em indivíduos amazônicos, visando a identificação de possíveis padrões específicos para a região, e posterior elaboração de uma chave de identificação para os morcegos da Amazônia Brasileira que contenha 
realmente informações úteis para a correta identificação destes taxa. Da mesma forma, reforçamos também a necessidade de que os acervos das coleções de referência presentes na região, tais como o INPA, o MPEG e o IEPA, precisam de cuidadosa revisão periódica, frente ao acréscimo de novas informações, publicações de novas chaves de identificação, e às constantes mudanças nomenclaturais experimentadas entre vários gêneros e espécies de morcegos.

\section{Técnicas de inventário}

A grande maioria dos inventários já conduzidos na Amazônia foi baseada principal ou exclusivamente no uso de redes de neblina, fator que contribui para a subestimativa da real diversidade de espécies de morcegos na região. O uso de redes é pouco eficaz na coleta de insetívoros aéreos que tendem a ser subamostrados com esta técnica (veja Simmons \& Voss 1998). De fato, algumas das espécies menos registradas e menos conhecidas para o bioma são insetívoros (e.g. Cytarops alecto, Diclidurus spp., Natalus espiritosantensis, Furipterus horrens, Eptesicus andinus, E. diminutus, Lasiurus spp., Rhogeessa io, Cynomops greenhalli, e Molossops neglectus).

Desta forma, um viés metodológico (uso predominante de redes) nos coloca diante do questionamento: estas são espécies intrinsicamente raras ou elas são, na verdade, mal amostradas? O uso de gravações de sinais de ecolocalização poderia contribuir significativamente para o registro de espécies pouco capturadas com redes (e.g. MacSwiney et al. 2008), especialmente os insetívoros aéreos, e inventários futuros na região amazônica devem priorizar o uso de métodos complementares de captura, incluindo a busca diurna de abrigos, de forma a melhor contribuir para o registro da parcela de espécies que se encontra mais mal amostrada.

\section{Agradecimentos}

Agradecemos ao Instituto Nacional de Pesquisas da Amazônia (INPA), ao Projeto Dinâmica Biológica de Fragmentos Florestais (PDBFF), à Elisabeth Kalko e Charles Handley (in memorian). E. Sampaio agradece ao Departamento de Zoofisiologia da Universidade de Tübingen e a Universidade de Ulm (ambas na Alemanha), CAPES/ DAAD e WWF-Brasil. E. Bernard agradece ao Departamento de Zoologia da UFPE, ao CNPq, Bat Conservation International, Smithsonian Institution, Capes, Conservação Internacional, M. Brock Fenton, Bill Magnusson, Ana Albernaz, Edvaldo Faria e Adnor Costa. V. Tavares agradece ao departamento de Zoologia da Universidade Federal de Minas Gerais por acolhê-la como pesquisadora associada, e a Gustavo Fonseca, Sueli Marques-Aguiar, Maria Nazareth Ferreira da Silva, Nancy B. Simmons, Linda Gordon, Alfred L. Gardner e Paul M. Velazco. Os autores agradecem a Renato Gregorin, Alexandre Percequillo e dois revisores anônimos, por vários comentários enriquecedores ao trabalho. Da mesma forma, agradecemos a todos aqueles que geraram os registros utilizados nesta compilação.

\section{Referências Bibliográficas}

ALBERICO, M., CADENA, A., HERNÁNDEZ-CAMACHO, J. \& MUÑOZSABA, Y. 2000. Mamíferos (Synapsida: Theria) de Colombia. Biota Colomb. 1:43-75. $\{1\}$

ALLEN, J.A. 1916. Mammals collected on the Roosevelt Brazilian Expedition, with field notes by Leo E. Miller. Bul. Am. Mus. Nat. Hist. 35:559-610. \{2\}

ASCORRA, C.F., WILSON, D.E. \& HANDLEY Jr., C.O. 1991. Geographic distribution of Molossops neglectus Williams and Genoways (Chiroptera: Molossidae). J. Mam. 72:828-830. \{3\}

BAKER, R.J., HOOFER, S.R., PORTER, C.A. \& VAN DEN BUSSCHE, R.A. 2003. Diversification among New World leaf-nosed bats: an evolutionary hypothesis and classification inferred from digenomic congruence of DNA sequence. Occas. Pap., Mus. Texas Tech Univ. 230:1-32. \{4\}
BERNARD, E. 1999. Notes on a colony of Peropteryx leucoptera (Emballonuridae) in Brazil. Bat Res. News 40(2):37-38. \{5\}

BERNARD, E. 2001a. First capture of Micronycteris homezi Pirlot (Chiroptera: Phyllostomidae) in Brazil. Revta Bras. Zool. 18(2): 645-647. \{6\}

BERNARD, E. 2001b. Species list of bats (Mammalia: Chiroptera) of Santarém area, Pará State, Brazil. Revta Bras. Zool. 18:455-463. \{7\}

BERNARD, E. 2001c. Vertical stratification of bat communities in primary forests of Central Amazon, Brazil. J. Trop. Ecol. 17(1):115-126. \{8\}

BERNARD, E. \& FENTON, M.B. 2002. Species diversity of bats (Chiroptera: Mammalia) in forest fragments, primary forests and savannas in Central Amazonia, Brazil. Can. J. Zool. 80:1124-1140. \{9\}

BERNARD E. \& SAMPAIO, E.M. 2008. Morcegos da Amazônia Brasileira. In Morcegos no Brasil: biologia, sistemática, ecologia e conservação (S.M Pacheco, R.V. Marques \& C.E.L. Esbérard, eds). Armazém Digital, Porto Alegre, p. 243-252. $\{10\}$

BERNARD, E., ALBERNAZ, A.L.K.M. \& MAGNUSSON, W.E. 2001. Bat species composition in three localities in the Amazon basin. Stud. Neotrop. Fauna Environ. 36:177-184. \{11\}

BERNARD, E., MACHADO, R.B. \& AGUIAR, L.M.S. 2011. Discovering the Brazilian bat fauna: a task for two centuries? Mammal Rev. 41(1):23-39. \{12\}

BEST, T.L., HUNT, J.L., MCWILLIAMS, L.A. \&. SMITH, K.G. 2001. Eumops maurus. Mamm. Species 667:1-3. \{13\}

BEZERRA, A.M.R. \& CUNHA, A.S. 2007. First occurrence of the genus Diclidurus Wied, 1820 (Emballonuridae: Emballonurinae) in central Brazil. Chirop. Neotrop. 13(1):205-306. $\{14\}$

CALENTANO, D. \& VERÍSSIMO, A. 2007. O avanço da fronteira na Amazônia: do boom ao colapso. O Estado da Amazônia Indicadores 2:1-46. $\{15\}$

CARTER, D.C. \& DOLAN, P.G. 1978. Catalogue of type specimens of Neotropical bats in selected European museums. Spec. Publ. Mus. Texas Tech Univ. 15:11-135. $\{16\}$

CARVALHO, C.T. 1960. Sobre alguns mamíferos do sudeste do Pará. Arq. Zool. Est. S. Paulo 11:121-132. $\{17\}$

CARVALHO, C.T. 1962. Lista preliminar dos mamíferos do Amapá. Pap. Av. Depto. Zool., São Paulo 15:283-297. \{18\}

CARVALHO, C.T. 1965. Comentários sobre alguns mamíferos descritos e figurados por Alexandre Rodrigues Ferreira em 1790. Arq. em Zoologia 12:7-70. $\{19\}$

CARVALHO, C.T. \& TOCCHETON, A.J. 1969. Mamíferos do Nordeste do Pará, Brasil. Rev. Biol. Tropical, 15(2): 215-226. \{20\}

CATZEFLIS, F. 2010. Liste des mammiferes de Guyane. Internal Report. University of Montpellier, France. $\{21\}$

CEBALlOS, G. \& MEDELLIN, R.A. 1988. Diclidurus albus. Mamm. Species 316:1-4. $\{22\}$

DALPONTE, J.C. \& AGUIAR, L.M.S. 2009. The first record of Diclidurus ingens Hernandez Camacho, 1955 (Emballonuridae) in Central Brazil. Biota Neotrop. 9(4):249-252. \{23\}

DAVIS, W.B. 1966. Review of South American bats of the genus Eptesicus. Southwest. Nat. 11:245-274. $\{24\}$

DAVIS, W.B. 1968. A review of the genus Uroderma (Chiroptera). J. Mam. 49:676-98. $\{25\}$

DISCHER, D.S., BERNARDE, P.S. \& FACURE, K.G. 2009. Mammalia, Chiroptera, Phyllostomidae, Vampyrum spectrum (Linnaeus, 1758): First record for the state of Rondônia, Brazil, and new prey records. CheckList 5(3):394-395. $\{26\}$

DOLAN, P.G. 1989. Systematics of Middle American mastiff bats of the genus Molossus. Spec. Publ. Mus. Texas Tech Univ. 29:1-71. \{27\}

EGER, J.L. 1977. Systematics of the genus Eumops (Chiroptera: Molossidae): Life Sci. Contr. R. Ont. Mus. 110:1-69. \{28\}

Eger, J.L. 2008. Family Molossidae. In Mammals of South America. Marsupials, xenarthrans, shrews, and bats. (Gardner, A.L., ed.).The University of Chicago Press, Chicago, p. 399-436.

FARIA, D., SOARES-SANTOS, B. \& SAMPAIO, E. 2006. Bats from the Atlantic rainforest of southern Bahia, Brazil. Biota Neotrop. 6:2-13. \{29\}

FERRELL C.S. \& WILSON, D.E. 1991. Platyrrhinus helleri. Mamm. Species 373:1-5. $\{30\}$

GARDNER, A.L. 2008. Mammals of South America. Marsupials, xenarthrans, shrews, and bats. The University of Chicago Press, Chicago, v.1. $\{31\}$ 
GENOWAYS, H.H. \& BAKER, R.J. 1996. A new species of the genus Rhogeessa, with comments on geographic distribution and speciation in the genus. In Contributions in mammalogy: a memorial volume honoring Dr. J. Knox Jones, Jr. (H.H. Genoways \& R.J. Baker, eds.). Museum of Texas Tech University, Lubock, p.83-87. \{32\}

GEORGE, T.K., MARQUES, S.A., de VIVO, M., BRANCH, L.C., GOMES, N. \& RODRIGUEZ, R. 1988. Levantamento de mamíferos do Parque Nacional da Amazonia (Tapajós). Brasil Florestal 63:33-41. \{33\}

GERVAIS, P. 1856. Documents pour servier à la monographie de chéiroptères Sud-Americaines. Comptes Rendus Acad. Sci. Paris 42:547-50. \{34\}

GIANNINI, N.P. \& BARQUEZ, R.M. 2003. Sturnira erythromos. Mamm. Species 729:1-5. $\{35\}$

GONÇALVES E. \& GREGORIN, R. 2004. Quirópteros da Estação Ecológica da Serra das Araras, Mato Grosso, Brasil, com o primeiro registro de Artibeus gnomus e A. anderseni para o Cerrado. Lundiana 5:143-149. \{36\}

GREGORIN, R. 1998. Extending geographic distribution of Chiroderma doriae Thomas, 1891 (Phyllostomidae, Stenodermatinae). Chirop. Neotrop. 4:98-99. $\{37\}$

GREGORIN, R. 2000. Filogenia de Molossidae Gervais, 1855 (Mammalia, Chiroptera). Tese de Doutorado, Universidade de São Paulo, São Paulo. \{38\}

GREGORIN, R. \& DITCHFIELD, A.D. 2005. New genus and species of nectar-feeding bat in the tribe Lonchophyllini (Phyllostomidae: Glossophaginae) from northeastern Brazil. J. Mammal. 86:403-14. \{39\}

GREGORIN, R. \& TADDEI, V.A. 2000. New records of Molossus and Promops from Brazil (Chiroptera: Molossidae). Mammalia 64:471-76. $\{40\}$

GREGORIN, R. \& TADDEI, V.A. 2002. Chave artificial para a identificação de Molossídeos brasileiros (Mammalia, Chiroptera). J. Neotrop. Mammal. 9(1):13-32. $\{41\}$

GREGORIN, R., CAPUSSO, G.L. \& FURTADO, V.R. 2008. Geographic distribution and morphological variation in Mimon bennettii (Chiroptera, Phyllostomidae). Iheringia, Sér. Zool. 98(3):404-411. \{42\}

HANDLEY, C.O. 1955. A new species of Free-tailed bat (genus Eumops) from Brazil. Proc. Biol. Soc. Wash. 68:177-178. \{43\}

HANDLEY, C.O. 1960. Descriptions of new bats from Panama. Proc. U.S. Nat. Mus. 3442:459-479. \{44\}

HANDLEY, C.O. 1967. Bats of the canopy of an Amazonian forest. Atas do Simpósio da Biota Amazônica 5:211-215. \{45\}

HANDLEY, C.O. 1987. New species of mammals from northern South America: fruit-eating bats, genus Artibeus Leach. Fieldiana Zool. 39:163-72. $\{46\}$

HANDLEY, C.O. Jr. \& FERRIS, K.C. 1972. Descriptions of new bats of the genus Vampyrops. Proc. Biol. Soc. Wash. 84:519-24. $\{47\}$

HANDLEY, C.O. Jr. \& OCHOA G., J. 1997. New species of mammals from northern South America: a sword-nosed bat, genus Lonchorhina Tomes (Chiroptera: Phyllostomidae). Mem. Soc. Cien. Nat. La Salle 57:71-82. $\{48\}$

HANSEN, M.C., STEHMAN, S.V., POTAPOV, P.V., LOVELAND, T.R., TOWNSHEND, J.R.G., DeFRIES, R.S., PITTMAN, K.W., ARUNARWATI, B., STOLlE, F., STEININGER, M.K., CARROLL, M. \& DiMICELI, C. 2008. Humid tropical forest clearing from 2000 to 2005 quantified by using multitemporal and multiresolution remotely sensed data. PNAS 105(27):9439-9444. $\{49\}$

HOOD, C. \& GARDNER, A.L. 2008. Family Emballonuridae Gervais, 1856. In Mammals of South America, marsupials, xenarthrans, shrews, and bats (A.L. Gardner, ed.). The University of Chicago Press, Chicago, v.1, p.188-207. $\{50\}$

HOOFER, S.R. \& BAKER, R.J. 2006. Molecular systematics of Vampyressine bats (Phyllostomidae: Stenodermatinae) with comparison of direct and indirect surveys of mitochondrial DNA variation. Mol. Phylogenet. Evol. 39:424-438. $\{51\}$

INSTITUTO BRASILEIRO DE GEOGRAFIA E ESTATÍSTICA - IBGE. 2010. Estatísticas. http://www.ibge.gov.br (último acesso em 23/10/2010). \{52\}

JONES, J.K. Jr. \& HOOD, C.S. 1993. Synopsis of South American bats of the family Emballonuridae. Occas. Pap. Mus. Texas Tech Univ. 155:1-32. \{53\}

KALKO, E.K.V. \& HANDLEY, C.O. 2001. Neotropical bats in the canopy: diversity, community structure, and implications for conservation. Plant Ecol. 33:546-550. $\{54\}$

KINGSTON T. 2009. Analysis of species diversity of bat assemblages. In Ecological and behavioral methods for the study of bats (T.H. Kunz \& S. Parsons, eds.). The John Hopkins University Press, Baltimore, p. 195-215. $\{55\}$
KUNZ, T.H. \& FENTON, M.B. 2003. Bat ecology. The University of Chicago Press, Chicago. $\{56\}$

LASSIEUR, S. \& WILSON, D.E. 1989. Lonchorhina aurita. Mamm. Species $374: 1-4 .\{57\}$

LAURANCE, W.F., COCHRANE, M.A., BERGEN, S., FEARNSIDE, P.M., DELAMÔNICA, P., BARBER, C., D’ANGELO, S. \& FERNANDES, T. 2001. The future of the Brazilian Amazon. Science 291(5503):438439. $\{58\}$

LAVAL, R.K. 1973a. A revision of the Neotropical bats of the genus Myotis. Sci. Bull. Los Angeles Co. Mus. 15:1-54. $\{59\}$

LAVAL, R.K. 1973b. Systematics of the genus Rhogeessa (Chiroptera: Vespertilionidae). Occ. Pap. Mus. Nat. Hist. Univ. Kans. 19:1-47. \{60\}

LIM, B.K. \& ENGSTROM, M.D. 2001. Species diversity of bats (Mammalia: Chiroptera) in Iwokrama Forest, Guyana, and the Guianan subregion: implications for conservation. Biodiv. \& Cons. 10:613-657. \{61\}

LIM, B.K., ENGSTROM, M.D. \& OCHOA, J.G. 2005. Mammals. In Checklist of the terrestrial vertebrates of the Guiana Shield (T. Hollowell \& R.P. Reynolds, eds.). Bulletin of the Biological Society of Washington 13, p 77-92. $\{62\}$

LIM, B.K., ENGSTROM, M.D., LEE Jr., T.E., PATTON, J.C. \& BICKHAM, J.W. 2004. Molecular differentiation of large species of fruit-eating bats (Artibeus) and phylogenetic relationships based on the cytochrome $\mathrm{b}$ gene. Acta Chirop. 6:1-12. \{63\}

LIMA, J.L. 1926. Os morcegos da collecção do Museu Paulista. Rev. Mus. Paulista, São Paulo 14:43-128. \{64\}

LÓPEZ-GONZÁLEZ, C. \& PRESLEY, S.J. 2001. Taxonomic status of Molossus bondae J. A. Allen, 1904 (Chiroptera: Molossidae), with description of a new species. J. Mamm. 82(3):760-774. \{65\}

MACSWINEY, M.C, CLARKE, F.M. \& RACEY, P.A. 2008. What you see is not what you get: the role of ultrasonic detectors in increasing inventory completeness in Neotropical bat assemblages. J. Appl. Ecol. 45:1364-1371. $\{66\}$

MARCIENTE, R. \& CALOURO, A.M. 2009. Mammalia, Chiroptera, Phyllostomidae, Lampronycteris brachyotis (Dobson, 1879): first record in Acre, Brazil. Check List 5(4):886-889. \{67\}

MARINI, M.A. \& GARCIA, F.I. 2005. Conservação de aves no Brasil. Megadiversidade 1(1):95-102. \{68\}

MARQUES, S.A. 1985. Novos registros de morcegos do Parque Nacional da Amazônia (Tapajós), com observações do período de atividade noturna e reprodução. Bol. MPEG zool. 2:71-83. \{69\}

MARQUES, S.A. 1989. Ecologia animal. Levantamento faunístico da área sob influência da BR-364 (Cuiabá-Porto Velho). Programa Polonoroeste. SCT/PR CNPq, Programa Polonoreste, Relatório de Pesquisa n.4. \{70\}

MARQUES, S.A. \& OREN, D.C. 1987. First Brazilian record for Tonatia schulzi and Sturnira bidens (Chiroptera: Phyllostomidae). Bol. MPEG zool. 3:159-60. \{71\}

MARQUES-AGUIAR, S.A. \& AGUIAR, G.F.S. 2002. Interações de quirópteros em ecossistemas tropicais: perspectivas de estudo para Caxiuanã. In Caxiuanã: populações tradicionais, meio físico e diversidade biológica (P.L.B. Lisboa, org.). MPEG, Belém, p.651-668. \{72\}

MARQUES-AGUIAR, S.A., AGUILA, M.V., AGUIAR, G.F.S., SALDANHA, N., SILVA-JUNIOR, J.S. \& ROCHA, M.M.B. 2003. Caracterização e perspectivas de estudo dos quirópteros da Estação Científica Ferreira Penna- município de Melgaço- PA. Idéias e Debates 6:1-3. \{73\}

MARQUES-AGUIAR, S.A., MELO, C.C.S., AGUIAR, G.F.S. \& QUEIRÓZ, J.A.L. 2002. Levantamento preliminar da mastofauna da região de AnajásMuaná, Ilha de Marajó, Pará, Brasil. Rev. Bras. Zool., 19(3):841-854. \{74\}

MARTINS, A.C.M., BERNARD, E. \& GREGORIN, R. 2006. Inventários biológicos rápidos de morcegos (Mammalia, Chiroptera) em três unidades de conservação do Amapá, Brasil. Revta Bras. Zool. 23:1175-1184. \{75\}

MARTINS, A.C.M., BERNARD, E., GREGORIN, R. \& SILVA, W.A.S. 2011. Filling data gaps on the diversity and distribution of Amazonian bats (Chiroptera): The case of Amapá, easternmost Brazil. Zoologia. No prelo. $\{76\}$

MCLEELAN, L.J \& KOOPMAN, K.F. 2007. Sufamily Carolliinae Miller, 1924. In Mammals of South America, marsupials, xenarthrans, shrews, and bats (A.L. Gardner, ed.). The University of Chicago Press, Chicago, v. 1, p. $208-218 .\{77\}$

MCNAB, B.K. 1969. The economics of temperature regulation in Neotropical bats. Comp. Biochem. Physiol. 31:227-68. \{78\} 
MILLER, G.S. 1902. Twenty new American bats. Proc. Acad. Nat. Sci. Philadelphia 54:389 412. \{79\}

MILLER, G.S. 1913. Notes on the bats of the genus Molossus. Proc. U.S Nat. Mus. 46:85-92. $\{80\}$

MIRANDA RIBEIRO, A. 1914. Zoologia. Commisão de linhas telegráphicas estratégicas de Matto Grosso ao Amazonas. Annexo 5, Historia Natural. Publ. n.17, Mammíferos. 49p. $\{81\}$

MITTERMEIER, R.A., MITTERMEIER, C.G., GIL, P.R. \& PILGRIM, J. 2000. Wilderness. Earth's last wild places. CEMEX, Mexico City. $\{82\}$

MOK, W.Y. \& LACEY, L.A. 1980. Algumas considerações ecológicas sobre morcegos vampiros na epidemiologia da raiva humana na Bacia Amazônica. Acta Amaz. 10:335-42. \{83\}

MOK, W.Y., WILSON, D.E., LACEY, L.A. \& LUIZÃO, R.C.C. 1982. Lista atualizada de quirópteros da Amazônia brasileira. Acta Amaz. 12:81723. $\{84\}$

MORTON, D.C., DEFRIES, R.S., SHIMABUKURO, Y.E., ANDERSON, L.O., ARAI, E., ESPIRITO-SANTO, F.B., FREITAS, R. \& MORISETTE, J. 2006. Cropland expansion changes deforestation dynamics in the southern Brazilian Amazon. PNAS 103(39):14637-14641. \{85\}

NOGUEIRA, M.R., POL, A. \& PERACCHI, A.L. 1998. Use of water holes by Stenodermatinae bats in western Amazonian Brazil. Bat Res. News. 39:95. $\{86\}$

NOGUEIRA, M.R., POL, A. \& PERACCHI, A.L. 1999. New records of bats from Brazil with a list of additional species for the chiropteran fauna of the state of Acre, western Amazon basin. Mammalia 63:363-368. \{87\}

OCHOA, J.G. \& SÁNCHEZ, J.H. 2005. Taxonomic status of Micronycteris homezi (Chiroptera, Phyllostomidae). Mammalia 69:323-36. $\{88\}$

ORTEGA, J. \& ARITA, H.T. 1997. Mimon bennettii. Mamm. Species 549:1-4. $\{89\}$

OSGOOD, W.H. 1916. Mammals of the Collins-Day South American Expedition. Field Mus. Nat. Hist. Zool. ser. 10:199-216. \{90\}

PACHECO S.M., MARQUES, R.V. \& ESBÉRARD, C.E.L. 2008. Morcegos do Brasil: biologia, ecologia e conservação. Armazém Digital, Porto Alegre. $\{91\}$

PACHECO, V. \& PATTERSON, B.D. 1991. Phylogenetic relationships of the new world bat genus Sturnira (Chiroptera: Phyllostomidae). Bull. Am. Mus. Nat. Hist. 206:101-121. \{92\}

PAGLIA, A.P., FONSECA, G.A.B., RYLANDS, A.B., HERRMANN, G., AGUIAR, L.M.S., CHIARELLO, A.G., LEITE, Y.L.R., COSTA, L.P., SICILIANO, S., KIERULFF, C.M., MENDES, S.L., TAVARES, V., MITTERMEIER, R.A. \& PATTON, J.L. 2011. Anoted List of Brazilian Mammals. $2^{\text {nd }}$ version revised. Occasional Papers in Conservation Biology, The University of Chicago Press, Chicago. No prelo. $\{93\}$

PATTERSON, B.D. 1992. Mammals in the Royal Natural History Museum, Stockholm, collected in Brazil and Bolivia by A. M. Olalla during 19341938. Fieldiana Zool. 66:1-42. \{94\}

PERACCHI, A.L. 1986. Considerações sobre a distribuição e a localidadetipo de Sphaeronycteris toxophyllum Peters, 1882 (Chiroptera: Phyllostomidae). Pub. Av. MNRJ 97-100. \{95\}

PERACCHI, A.L. \& ALBURQUERQUE, S.T. 1985. Considerações sobre a distribuição geográfica de algumas espécies do gênero Micronycteris Gray, 1866 (Mammalia, Chiroptera, Phyllostomidae). Arq. UFRRJ 8:23-26. $\{96\}$

PERACCHI, A.L., RAIMUNDO, S.D.L. \& TANNURE, A.M. 1984. Quirópteros do território Federal do Amapá, Brasil (Mammalia: Chiroptera). Arq. UFRRJ 89-100. \{97\}

PETERS, S.L., LIM, B.K. \& ENGSTROM, M.D. 2002. Systematics of dogfaced bats (Cynomops) based on molecular and morphometric data. J. Mamm. 83:1097-1110. \{98\}

PICCININI, R.S. 1974. Lista provisoria dos quirópteros da colecão do Museu Paraense Emílio Goeldi (Chiroptera). Bol. MPEG zool. 77:1-32. \{99\}

PINE, R.H. 1993. A new species of Thyroptera Spix (Mammalia: Chiroptera: Thyropteridae) from the Amazon Basin of northeastern Perú. Mammalia 57:213-225. $\{100\}$

PINE, R.H., BISHOP, I.R. \& JACKSON, R.L. 1970. Preliminary list of mammals of the Xavantina/Cachimbo expedition (Central Brazil). Trans. Royal Soc. Trop. Med. Hyg. 64:668-670. \{101\}
PINE, R.H., LAVAL, R.K., CARTER, D.C. \& MOK, W.Y. 1996. Notes on the graybeard bat, Micronycteris daviesi (Hill) (Mammalia: Chiroptera: Phyllostomidae), with the first records from Ecuador and Brazil. In Contributions in Mammalogy: A Memorial Volume Honoring Dr. J. Knox Jones, Jr. (H.H. Genoways \& R.J. Baker, eds). Museum of Texas Tech University, Lobock, p.183-190. \{102\}

PIRLOT, P. 1972. Chiropteres de Moyenne Amazonie. Mammalia 36:71-85. $\{103\}$

PRESLEY, S.J., WILLIG, M.R., WUNDERLE Jr., M. \& SALDANHA, L.N. 2008. Effects of reduced-impact logging and forest physiognomy on bat populations of lowland Amazonian forest. J. Appl. Ecol. 45(1):14-25. \{104\}

REIS, N.R. 1984. Estrutura de comunidade de morcegos na região de Manaus, Amazonas. Rev. Bras. Biol. 44:247-54. \{105\}

REIS, N.R. \& GUILLAUMET, J.L. 1983. Les chauvessouris frugivores de la région de Manaus et leur rôle dans la dissémination des espèces véegétales. Rev. Ecol. (Terre Vie) 38:147-69. \{106\}

REIS, N.R. \& PERACCHI, A.L. 1987. Quirópteros da região de Manaus, Amazonas, Brasil (Mammalia, Chiroptera). Bol. MPEG zool. 3:161-82. $\{107\}$

REIS, N.R. \& SCHUBART, H.O.R. 1979. Notas preliminares sobre os morcegos do Parque Nacional da Amazônia (Médio Tapajós). Acta Amaz. 9:507-515. \{108\}

REIS, N.R., PERACCHI, A.L., PEDRO, W.A. \& LIMA, I.P. 2007. Morcegos do Brasil. Universidade de Londrina, Londrina, 253p. \{109\}

ROBINSON, F. 1998. The bats of the Ilha de Maracá. In Maracá: the biodiversity and environment of an Amazonian Rainforest (J.A. Ratter $\&$ W. Milliken, eds.). John Wiley \& Sons, London, p.165-188. $\{110\}$

RODRIGUES, A.S.L., EWERS, R.M., PARRY, L., SOUZA Jr., C., VERÍSSIMO, A. \& BALMFORD, A. 2009. Boom-and-bust development patterns across the Amazon deforestation frontier. Science 324(5933):1435-1437. $\{111\}$

RODRIGUES, M.T. 2005. Conservação dos répteis brasileiros: os desafios para um país megadiverso. Megadiversidade 1(1):87-94. \{112\}

SALDANHA, N.L. 2000. Caracterização da comunidade de quirópteros (Mammalia) em áreas naturais e manejadas da Floresta Nacional do Tapajós, PA - Brasil. Dissertação de Mestrado, Universidade Federal do Pará, Belém. $\{113\}$

SALDANHA, N.L., AGUIAR, S.M. \& ROCHA, M.B.B. 2000. Levantamento de quirópteros da Floresta Nacional do Tapajós. In XXIII Congresso Brasileiro de Zoologia. Universidade Federal de Mato Grosso, Cuiabá, p.587. $\{114\}$

SAMPAIO, E.M., KALKO, E.K.V., BERNARD, E., RODRÍGUEZHERRERA, B. \& HANDLEY, C.O. 2003. A biodiversity assessment of bats (Chiroptera) in a tropical lowland rainforest of Central Amazonia, including methodological and conservation considerations. Stud. Neotrop. Fauna Environ. 38(1):17-31. \{115\}

SANBORN, C.C. 1932. Neotropical bats in the Carnegie Museum. Annals of the Carnegie Museum 21:171-183. \{116\}

SANBORN, C.C. 1937. American bats of the subfamily Emballonuridae. Field Mus. Nat. Hist. Zool. Series 20:321-354. \{117\}

SANBORN, C.C. 1949. Bats of the genus Micronycteris and its subgenera. Fieldiana Zool. 31:215-233. \{118\}

SCHNEIDER, M.C., ROMIJN, P.C., UIEDA, W., TAMAYO, H., da SILVA, D.F., BELOTTO, A., da SILVA, J.B. \& LEANES, L.F. 2009. Rabies transmitted by vampire bats to humans: An emerging zoonotic disease in Latin America? Pan. Am. J. Public Health 25(3):260-269. \{119\}

SCHNEIDER, M.C., SANTOS-BORGOA, C., ARON, J., MUÑOZ, B., RUIZ-VELAZCO, S. \& UIEDA, W. 1996. Potential force of infection of human rabies transmitted by vampire bats in the Amazonian region of Brazil. Am. J. Trop. Med. Hyg. 5:680-684. \{120\}

SHUMP Jr., K.A. \& SHUMP, A.U. 1982. Lasiurus borealis. Mamm. Species 183:1-6. $\{121\}$

SILVA, M.N.F., RYLANDS, A.B. \& PATTON, J.L. 2001. Biogeografia e conservação da mastofauna na floresta amazônica brasileira. In Biodiversidade na Amazônia Brasileira: avaliação e ações prioritárias para a conservação, uso sustentável e repartição de benefícios. (J.P.R. Capobianco, A. Veríssimo, A. Moreira, D. Sawyer, I. dos Santos \& L.P. Pinto, orgs.). Instituto Socioambiental, São Paulo. $\{122\}$

SIMMONS, N.B. 1996. A new species of Micronycteris (Chiroptera: Phyllostomidae) from Northeastern Brazil, with comments on phylogenetic relationships. Am. Mus. Novit. 3158:1-34. \{123\} 
SIMMONS, N.B. 2005. Chiroptera. In Mammal species of the world: a taxonomic and geographic reference (D.E. Wilson \& D.M. Reeder, eds.). Johns Hopkins University Press, Baltimore. $\{124\}$

SIMMONS, N.B. \& VOSS, R.S. 1998. The mammals of Paracou French Guiana: a Neotropical lowland rainforest fauna. Part I. Bats. Bul. Am. Mus. Nat. Hist. 237: 1-219. $\{125\}$

SIMMONS, N.B., VOSS, R.S. \& FLECK, D.W. 2002. A new Amazonian species of Micronycteris (Chiroptera: Phyllostomidae) with notes on the roosting behavior of sympatric congeners. Am. Mus. Novit. 3358:1-14. $\{126\}$

SOLARI, S. \& BAKER, R.J. 2006. Mitochondrial DNA sequence, karyotypic, and morphological variation in the Carollia castanea species complex (Chiroptera: Phyllostomidae) with description of a new species. Occas. Papers Mus. Texas Univ. 254:1-16. \{127\}

TADDEI, V.A. \& REIS, N.R. 1980. Notas sobre alguns morcegos da Ilha de Maracá, Território Federal de Roraima (Mammalia, Chiroptera). Acta Amaz. 10:363-68. $\{128\}$

TADDEI, V.A. \& REIS, N.R. 1983. Uma nova espécie de Lonchophylla do Brasil e chave para identificação das espécies do gênero (Chiroptera, Phyllostomidae). Ciênc. Cult. 35:625-29. \{129\}

TADDEI, V.A. \& UIEDA, W. 2001. Distribution and morphometrics of Natalus stramineus from South America (Chiroptera, Natalidae). Iheringia, Sér. Zool. 91:123-132. \{130\}

TADDEI, V.A. \& VICENTE-TRANJAN, E.C. 1998. Biological and distributional notes on Platyrrhinus helleri (Chiroptera: Phyllostomidae) in Brazil. Mammalia 62:112-17. \{131\}

TADDEI, V.A., REZENDE, I.M. \& CAMORA, D. 1990. Notas sobre uma coleção de morcegos de Cruzeiro do Sul, Rio Juruá, Estado do Acre (Mammalia: Chiroptera). Bol. MPEG zool. 6:75-88. \{132\}

TADDEI, V.A., VIZOTTO, L.D. \& SAZIMA, I. 1978. Notas sobre Lionycteris e Lonchophylla nas colecções do Museu Paraense Emílio Goeldi (Mammalia, Chiroptera, Phyllostomidae). Bol. MPEG zool. 92:1-14. \{133\}

TAVARES, V.C., GREGORIN, R. \& PERACCHI, A.L. 2008. Diversidade de morcegos no Brasil: lista atualizada com comentários sobre distribuição e taxonomia. In Morcegos no Brasil: biologia, sistemática, ecologia e conservação (S.M. Pacheco, R.V. Marques, R.V. \& C.E.L. Esberard, orgs.). Armazém Digital, Porto Alegre, p. 25-58. \{134\}

TEJEDOR, A. 2006. The type locality of Natalus stramineus (Chiroptera: Natalidae): implications for the taxonomy and biogeography of the genus Natalus. Acta Chirop 8(2):361-380. \{135\}

TEJEDOR, A., TAVARES, V.C. \& SILVA-TABOADA, G. 2005. A revision of extant Greater Antillean bats of the genus Natalus (Chiroptera: Natalidae). Am. Mus. Novit. 3493:1-22. \{136\}

THOMAS, O. 1901a. A new free-tailed bat from the lower Amazons. Ann. Mag. Nat. Hist. ser. 7, 7:190-91. \{137\}

THOMAS, O. 1901b. New species of Saccopteryx, Sciurus, Rhipidomys and Tatu from South America. Ann. Mag. Nat. Hist. 7:366-371. \{138\}

THOMAS, O. 1901c. On a collection of bats from Para. Ann. Mag. Nat. Hist., ser. 7, 8:189-93. $\{139\}$

THOMAS, O. 1912. New bats and rodents from S. America. Ann. Mag. Nat. Hist. ser. 8, 10:403-411. $\{140\}$

THOMAS, O. 1913. On some rare Amazonian mammals from the collection of the Para Museum. Ann. Mag. Nat. Hist. ser. 8, 11:130-136. $\{141\}$

THOMAS, O. 1915. On bats of the genus Promops. Ann. Mag. Nat. Hist. 8:61-65. $\{142\}$

THOMAS, O. 1916. Notes on the bats of the genus Histiotus. Ann. Mag. Nat. Hist. ser. 9, 5: 188-196. \{143\}

THOMAS, O. 1920a. On Neotropical bats of the genus Eptesicus. Ann. Mag. Nat. Hist. ser. 9, 5:360-67. \{144\}

THOMAS, O. 1920b. On mammals from the lower Amazons in the Goeldi Museum, Para. Ann. Mag. Nat. Hist. ser. 9, 6:266-83. $\{145\}$

TRAJANO, E. \& MOREIRA, J.R.A. 1991. Estudo da fauna de cavernas da Província Espeleológica Arenítica Altamira-Itaituba, Pará. Rev. Brasil. Biol. 51:13-29. $\{146\}$

UIEDA, W. 1980. Ocorrência de Carollia castanea na Amazônia Brasileira (Chiroptera, Phyllostomidae). Acta Amaz. 10:936-938. \{147\}
UIEDA, W. \& PEDRO, W.A. 1996. Chiroptera in the XXI Brazilian Zoology Congress. Chiropt. Neotrop. 2:41-42. \{148\}

VELAZCO, P.M. 2005. Morphological phylogeny of the bat genus Platyrrhinus Saussure, 1860 (Chiroptera: Phyllostomidae) with the description of four new species. Fieldiana Zool. 105:1-53. \{149\}

VELAZCO, P.M. \& GARDNER, A.L. 2009. A new species of Platyrrhinus (Chiroptera: Phyllostomidae) from western Colombia and Ecuador, with emended diagnoses of P. aquilus, P. dorsalis, and P. umbratus. Proc. Biol. Soc. Wash. 122(3):249-281. $\{150\}$

VELAZCO, P.M. \& PATTERSON, B.D. 2008. Phylogenetics and biogeography of the broad-nosed bats, genus Platyrrhinus (Chiroptera: Phyllostomidae). Mol. Phyl. Evol. 49:749-759. \{151\}

VELAZCO, P.M., GARDNER, A.L. \& PATTERSON, B.D. 2010. Systematics of the Platyrrhinus helleri complex (Chiroptera: Phyllostomidae), with descriptions of two new species. Zool. J. Linnean Soc. 159:785-812. \{152\}

VIEIRA, C.O.C. 1942. Ensaio monográfico sobre os quirópteros do Brasil. Arq. Zool. Est. S. Paulo 3:1-47. \{153\}

VIEIRA, C.O.C. 1948. Nova contribuição ao conhecimento dos mamíferos do Rio Juruá. Bol. MPEG zool. 10:239-274. \{154\}

VIEIRA, C.O.C. 1951. Notas sobre mamíferos obtidos pela expedição do Instituto Butantan ao Rio das Mortes e Serra do Roncador. Pap. Av. Zool. MZUSP 10:105-125. \{155\}

VIEIRA, C.O.C. 1955. Lista remissiva dos mamíferos do Brasil. Arq. Zool. Est. S. Paulo 8:341-474. $\{156\}$

VIEIRA, C.O.C. 1957. Sobre mamíferos do estado do Maranhão. Pap. Av. Zool. MZUSP 13:125-132. $\{157\}$

VIZOTTO, L.D. \& TADDEI, V.A. 1976. Notas sobre Mollosops temminckii temminckii e Mollosops planirostris (Chiroptera - Molossidae). Naturalia 2:47-59. $\{158\}$

VOSS, R.S. \& EMMONS, L.H. 1996. Mammalian diversity in Neotropical lowland rainforests: a preliminary assessment. Bul. Am. Mus. Nat. Hist. 230:1-115. $\{159\}$

WAGNER, J.A. 1843. Diagnosen neuer Arten Brasilischer Handflügler. Arch. Naturgesch. 9:365-368. $\{160\}$

WEBSTER, W.D. 1993. Systematics and evolution of bats of the genus Glossophaga. Spec. Pub. Mus. Texas Tech. Univ. 36:1-184. \{161\}

WEBSTER, W.D. \& HANDLEY. C.O. 1986. Systematics of Miller's long tongued bat, Glossophaga longirostris, with description of two new species. Occ. Pap. Mus. Texas Tech Univ. 100:1-22. \{162\}

WEBSTER, W.D \& JONES Jr., J.K. 1987. A new subspecies of Glossophaga commissarisi (Chiroptera: Phyllostomidae) from South America. Occ. Pap. Mus. Texas Tech Univ. 109:1-7. \{163\}

WEBSTER, W.D. \& JONES Jr., J.K. 1993. Glossophaga commissarisi. Mamm. Species 446:1-4. $\{164\}$

WILLIAMS, C.F. 1978. Taxonomic and karyologic comments on small brown bats, genus Eptesicus, from South America. Ann. Carnegie Mus. 47:361-383. $\{165\}$

WILLIAMS, S.L. \& GENOWAYS, H.H. 1980. Results of the Alcoa FoundationSuriname Expeditions. IV. A new species of bat of the genus Molossops (Mammalia: Molossidae). Ann. Carnegie Mus. 49:487-498. \{166\}

WILLIAMS, S.L., PHILLIPS, C.J. \& PUMO, D.E. 1990. New records of bats from French Guiana. Texas J. Sci. 42:204-206. $\{167\}$

WILLIAMS, S.L., WILLIG, M.R. \& REID, F.A. 1995. Review of the Tonatia bidens complex (Mammalia: Chiroptera), with descriptions of two new subspecies. J. Mamm. 76:612-626. \{168\}

WILSON, D.E. \& REEDER D.M. 2005. Mammal species of the world: a taxonomic and geographic reference. Johns Hopkins University Press, Baltimore. $\{169\}$ 